Supporting Information

\title{
Pulsed Saturated Absorption Competition Microscopy on Nonbleaching Nanoparticles
}

Chuankang $\mathrm{Li}^{1}$, Yuhang $\mathrm{Li}^{1}$, Yubing Han ${ }^{1}$, Zhimin Zhang ${ }^{1}$, Yuzhu Li ${ }^{1}$, Wensheng Wang $^{1}$, Xiang Hao ${ }^{1}$, Cuifang Kuang ${ }^{1,2,3, *}$, and $\mathrm{Xu} \mathrm{Liu}^{1,3}$

${ }^{1}$ State Key Laboratory of Modern Optical Instrumentation, College of Optical Science and Engineering, Zhejiang University, Hangzhou, Zhejiang 310027, China

${ }^{2}$ Ningbo Research Institute, Zhejiang University, Ningbo 315100, China

${ }^{3}$ Collaborative Innovation Center of Extreme Optics, Shanxi University, Taiyuan, Shanxi 030006, China

*cfkuang@zju.edu.cn

SUPPLEMENTARY TEXT.

1.Experimental setup ...............................................

2.Impacts ........................................................... 3

3.Supplementary imaging results .................................... 3

4. Sample preparation.................................................. 3

5. Data processing .................................................. 4

6. Theory …...................................................................

SUPPLEMENTARY FIGURES AND TABLE.......................9

REFERENCES ...............................................................26 


\section{Supporting Information}

\section{SUPPLEMENTARY TEXT}

\section{Experimental setup}

In the previous study of SAC, using continuous wave $(\mathrm{CW})$ lasers, the unrejected signal of the doughnut saturation beam, together with the amplified Poisson noise, routinely composes the dominate noise in SAC system under non-detector saturation condition. When saturation intensity is too high, the accompanied noise affect the accuracy of the readout image. Thus, imaging of much more complex biological structures, like microtubules and mitochondria, becomes undesirable. Pulsed laser should be a solution to the trade-off of the SAC's resolution with the accompanied noise. Compared with CW SAC, pSAC could achieve relatively high resolution with high SNR under the same doughnut saturation beam power. The instantaneous saturated competition beam (hollow beam) intensity in the CW scheme is typically lower, and so is the instantaneous probability of inhibition de-excitation. A non-negligible part of the molecules still emits fluorescence because they have not been exposed to sufficient de-exciting photons. Such fluorescence is particularly prevalent right at the slopes of the zero-intensity point of the hollow beam where the hollow beam is weaker. While, in the pulsed mode, all the photons act shortly after the excitation event and relatively high instantaneous inhibition rate is guaranteed. ${ }^{1}$ To this end, the less efficient spatial confinement of fluorescence associated with $\mathrm{CW}$-mode SAC is disadvantageous compared to pulsed SAC.

The experimental scheme of pulsed saturated competition microscopy (pSAC) is shown in Figure S1. A pulsed beam of $532 \mathrm{~nm}$ wavelength is collimated by collimation lens (CL1). A polarizing beam splitter (PBS1) and a half wave plate (HWP) are utilized to generate $S$-polarized beam which meets the polarization requirement of the acoustic-optic modulator (AOM). The incident beam is split into $P$-polarized and $S$-polarized beams. The former is spatially modulated by a vortex phase plate (VPP) to obtain doughnut-shaped beam (hollow beam, $I_{\text {hexc }}$ ). The latter is time-modulated with certain frequency which remains Gauss-shaped profile (solid beam, $I_{\text {exc }}$ ) through AOM based on Bragg diffraction principle. What's more, the two pulse beams have to be overlapped in spatial and temporal domain. And the scheme of optical time delay is utilized to achieve the precise temporal overlapping. The galvanometer-based scanning mirrors (GM) realize the two-dimensional fast scanning of focal beams on the sample plane. The 1.4 NA oil immersion objective lens (OL) focuses the illumination beams and collects the fluorescence emission simultaneously. The dichroic mirror (DM) is used to transmit illumination beams and reflect fluorescence signals. Finally, focused by the converging lens (CL2) and filtered by a pinhole $(0.5 \mathrm{AU})$, the fluorescence signals are detected via the photomultiplier tube (PMT). In our work, homodyne detection scheme is employed using the lock-in amplifier (LIA) to separate the wanted fluorescence $\left(F_{\text {exc }}\right)$ excited merely by the solid beam $\left(I_{\text {exc }}\right)$ radiation with certain frequency. A radio frequency driver (RF) is for generation of ultrasonic waves so that the refractive index of the acoustic-optic crystal in AOM could change periodically. The function generator (FG) offers the same reference frequency for both LIA and RF. Indeed, fluorescence signals $\left(F_{\text {hexc }}\right)$ excited by the hollow beam $\left(I_{\text {hexc }}\right)$ is deemed as background signals which are independent of the solid beam $\left(I_{e x c}\right)$ and consequently are discarded. While, for imaging non-fluorescent agents with scattering signals, like gold nanospheres, the only modification of the experimental setup is the removal of the fluorescent filter (F) so that the scattered plasmonic signals are received by the detector. 


\section{Supporting Information}

\section{Impacts}

Crucial impacts that would have great influence on the imaging results of pSAC are researched numerically and experimentally. These investigations will help to deeply understand the working principle of pSAC and to make full use of this novel super-resolved approach in extensive fields. Obviously, by competition of two kinds of signal elements, pSAC offers the guidance in how to extract weak component from the mixtures. Not merely confined in biological imaging (either fluorescent or non-fluorescent), in our perspective, $\mathrm{pSAC}$ would also be of great inspiration to much more utilizations, like physical science. The study focuses on the influences of illumination intensity (Figure S2), photobleaching (Figure S3, S4), dark spot central imperfection (Figure S2), pulse width (Figure S9, S10), as well as the spatial deviation (Figure S5, S6), wavelength mismatch (Figure S7, S8), and temporal displacement (Figure S9, S10) of the two beams. Simulations in Figure S2, S3, S5 and S7 are implemented with $I_{\text {exc }} 10 \mathrm{~kW} / \mathrm{cm}^{2}$ and $I_{\text {hexc }} 900 \mathrm{~kW} / \mathrm{cm}^{2} . F_{\text {exc }}$ denotes fluorescence emission by $I_{e x c}$ and $F_{\text {hexc }}$ denotes fluorescence emitting from $I_{\text {hexc }}$. Poisson noise caused by saturation detection is ignored in all simulations of Figure S2, S4, S9 and S10.

\section{Supplementary imaging results}

As we know, pSAC super-resolution microscopy, as one of STED derivatives, features easy-to-implement optics with moderate illumination power and improved signal-to-background ratio (SBR). And pSAC is also potential in the imaging of several kinds of nanoparticles, like gold nanoparticles (GNPs), fluorescent nanodiamonds (FNDs) and quantum dots (QDs). Furthermore, its utilization in biological observation verifies that $\mathrm{pSAC}$ is suitable for investigation of imaging sub-100-nanometer structures and organelles. Theoretical and experimental works have shown that pSAC can achieve the same spatial resolution with $\sim 20$ times lower light doses and 1.2 times higher SBR in fixed cell imaging of fluorescent nanodiamonds (FNDs) compared with STED. Note that the sub-diffraction size of the color centers (embedded in FNDs) is $\sim 60 \mathrm{~nm}$ which is slightly larger than the actual size of the FNDs ( $\sim 40 \mathrm{~nm}$, see Figure S12c). The differential SAC (dSAC) numerical algorithm is developed to further increase the attainable resolution (see Figure S12f). ${ }^{2}$, 3 Figure S11a-c suggests that pSAC might not work well on overlapping features (highlighted by the yellow arrow), in which case two or more particles, within the diffraction-limited range, may be included. Previous reports have shown that resolution improvement will be limited in mixed particles because of signal coupling between adjacent nanoparticles. This issue can be mitigated either by coating particles with shells of particular thicknesses or by properly tailoring particle surfaces to eliminate aggregation. ${ }^{4}$

\section{Sample preparation}

Cell Culture and Immunostaining. U2OS (human osteosarcoma cell line) cells were purchased from the American Type Culture Collection, and cultured in McCoy's 5A medium (Thermo Fisher Scientific, Inc.) supplemented with 10\% (v/v) FBS. The cultures were maintained at $37^{\circ} \mathrm{C}$ in a humidified $5 \% \mathrm{CO}_{2}$ environment. For FNDs, U2OS cells were seeded in standard conditions in Nunc Glass Bottom Dishes at a density of $1.5 \sim 2.0 \times 10^{4}$ per well in growth mediam $(150 \mu \mathrm{L})$. After overnight incubation, the cells were washed three times with phosphate buffer saline (PBS; Thermo Fisher Scientific, Inc.). The cells were incubated for 1.5 hour with FNDs at a 


\section{Supporting Information}

concentration of $10 \mu \mathrm{g} / \mathrm{mL}$ and then incubated for $30 \mathrm{~min}$ also with Mitotracker Deep Red at $1.5 \mu \mathrm{M}$. After incubation, the excess of dyes and FNDs were washed three times with solution. The cells were then fixed with 4\% paraformaldehyde in PBS and washed three times with PBS before observation.

For QDs, U2OS cells were seeded in Nunc Glass Bottom Dishes for overnight growth. Before staining, cells were washed with PBS for three times. Then the cells were fixed in 3\% paraformaldehyde and $0.1 \%$ glutaraldehyde in PBS for $10 \mathrm{~min}$. The cells were blocked and permeabilized with $5 \%$ goat serum and $0.2 \% \mathrm{v} / \mathrm{v}$ Triton X-100 in PBS for 1 hour. After blocking and permeabilization, the cells were labeled with Alpha-Tubulin Mouse monoclonal Antibody and TOMM20 Rabbit monoclonal Antibody at $1 \mu \mathrm{g} / \mathrm{mL}$ and incubated overnight at $4^{\circ} \mathrm{C}$. After washing with PBS, cells were stained for $60 \mathrm{~min}$ with two kinds of secondary antibodies conjugated with two kinds of quantum dots purchased from Thermo Fisher: Qdot565 Goat F(AB)2 Anti-mouse $\operatorname{IgG}$ conjugate $(\mathrm{H}+\mathrm{L})$ and Qdot605 Goat $\mathrm{F}(\mathrm{AB}) 2$ Anti-rabbit $\operatorname{IgG}$ conjugate $(\mathrm{H}+\mathrm{L})$. Then the cells were washed three times with PBS before imaging.

Nanoparticle Preparation. Fluorescent microspheres diluted in absolute ethyl alcohol are incubated on coverslips coated with poly L-lysine for 5 minutes. Then the samples are all mounted on microscope slides with Prolong Diamond Antifade Mountant (Thermo Fisher). Diluting ratio for F8800, F8801, N21483 nanoparticles (Thermo Fisher) is 1:500 while the counterpart for F8770 nanoparticles (Thermo Fisher) is 1:100. Gold nanoparticles stabilized by citrate were purchased from XFNANO Materials Co., Ltd. The particles were immobilized onto the silane-functionalized glass coverslips, and immersed in oil (refractive index 1.52), which matched the refractive index of the microscope slide to avoid strong reflection from the glass surface and finally the particles were sealed by a cover glass.Fluorescent nanodiamonds were ordered from Adamas Nanotechnologies, Inc. The particles were dried at a $0.01 \mathrm{mg} / \mathrm{mL}$ on a glass slide for photophysical properties characterization.

\section{Data processing}

Data analysis and numerical works were performed with MATLAB and ImageJ. The Gaussian fitting was done by utilizing a plugin from ImageJ-SMLM. The 3D images were generated using MATLAB. Color channel merging was performed by ImageJ.

\section{Theory}

Fluorescence Absorption Saturation. As for organic dye molecules, according to the five-level molecular model, shown in Figure $2 \mathrm{~d}$, rate equation system is established as follows: ${ }^{5}$

$$
\begin{gathered}
S_{0}=-k_{01} S_{0}+k_{f} S_{1}+k_{T} T_{1} \\
S_{1}=k_{01} S_{0}-\left(k_{0}+k_{1 n}+k_{b S}\right) S_{1}+k_{n 1} S_{n} \\
S_{n}=k_{1 n} S_{1}-\left(k_{n 1}+k_{b S n}\right) S_{n} \\
T_{1}=k_{I S C} S_{1}-\left(k_{T}+k_{T 1 n}+k_{b T}\right) T_{1}+k_{T n 1} T_{n} \\
T_{n}=k_{T 1 n} T_{1}-\left(k_{T n 1}+k_{b T n}\right) T_{n}
\end{gathered}
$$

$S_{0}, S_{1}$ and $S_{n}$ represent for singlet electronic populations of ground state, excited state and higher excited state, respectively; $T_{1}$ and $T_{n}$ represent for populations of triplet state and higher triplet state, respectively. And $k_{()}$represents for the rate constant in 


\section{Supporting Information}

each electronic level. Meanwhile, $k_{01}, k_{1 n}$ and $k_{T 1 n}$ are excitation rate constants in $S_{0}$, $S_{1}$ and $T_{1}$, respectively. As for de-excitation processes, $k_{f}, k_{S n l}, k_{T n l}$ and $k_{T}$ are depopulation rate constants in $S_{l}, S_{n}, T_{n}$ and $T_{l}$, respectively. And $k_{f}$ is also accounted as spontaneous fluorescence rate. And $k_{I S C}$ denotes the intersystem crossing rate constant; $k_{b}$ denotes the bleaching rate constant in corresponding level. For example, $k_{b S n}$ and $k_{b S}$ represent for the bleaching rate constants in $S_{n}$ and $S_{l}$, respectively. What's more, $\left.k_{(}\right)$is proportional to the applied radiation intensity and the corresponding absorption cross-section at the excitation wavelength as shown in Equation (2):

$$
k_{0}=\sigma I \lambda / h c
$$

Where $h$ is Planck's constant and $c$ is the velocity of light. The following parameters are values of rate constant and cross-section using the photophysical parameters of Rhodamine 6G with applied laser wavelength $515 \mathrm{~nm}: k_{0}=2.56 \times 10^{8} \mathrm{~s}^{-1} ; k_{f}=2.4 \times 10^{8}$ $\mathrm{s}^{-1} ; k_{I S C}=1.1 \times 10^{6} \mathrm{~s}^{-1} ; k_{T}=4.9 \times 10^{5} \mathrm{~s}^{-1} ; S_{n I}=5 \times 10^{12} \mathrm{~s}^{-1} ; k_{T n I}=5 \times 10^{12} \mathrm{~s}^{-1} ; k_{b}=650 \mathrm{~s}^{-1}$; $k_{b S n}=2.8 \times 10^{8} \quad \mathrm{~s}^{-1} ; \quad k_{b T n}=2.8 \times 10^{8} \quad \mathrm{~s}^{-1} ; \quad \sigma_{01}=2.7 \times 10^{-16} \quad \mathrm{~cm}^{2} ; \quad \sigma_{l n}=0.77 \times 10^{-17} \quad \mathrm{~cm}^{2} ;$ $\sigma_{T I n}=3.85 \times 10^{-17} \mathrm{~cm}^{2}$.

The following assumption should be considered. Intersystem crossing between $S_{n}$ and $T_{n}$, as well as stimulated emission and internal conversion between $S_{1}$ and $S_{0}$, is negligible. Furthermore, only the lowest vibronic level of each electronic state is considered, that is, fast relaxation from higher vibronic states is assumed since these transition processes are all slower than sub-picosecond relaxation as long as picosecond pulses are employed. After nanosecond or microsecond equilibrium, the steady-state approximation is reached in case of $S_{0}+S_{1}+S_{n}+T_{1}+T_{n}=1$, given in population probability equations as follows:

$$
\begin{gathered}
S_{0 e q}=k_{T n 1} k_{S n 1} k_{T} k_{0} / X \\
X=k_{T n 1}\left[k_{T}\left(k_{S n 1}\left(k_{0}+k_{01}\right)+k_{01} k_{1 n}\right)\right]+\left(k_{T n 1}+k_{T 1 n}\right)\left(k_{I S C} k_{S n 1} k_{01}\right) \\
S_{1 e q}=S_{0 e q} k_{01} / k_{0} \\
S_{n e q}=S_{1 e q} k_{1 n} / k_{S n 1} \\
T_{1 e q}=S_{1 e q} k_{I S C} / k_{T} \\
T_{n e q}=k-T_{1 e q} k_{T 1 n} / k_{T n 1}
\end{gathered}
$$

Five-level saturation equation is expressed as follows:

$$
S_{\text {leq_5level }}=k_{T n 1} k_{\text {Sn1 }} k_{T} k_{01} / X
$$

Four-level saturation equation is expressed as follows, with $k_{T n}=1 \mathrm{~s}^{-1}$ and $k_{T 1 n}=0$ :

$$
S_{\text {leq_4level }}=k_{S n 1} k_{T} k_{01} /\left[k_{T}\left(k_{S n 1} k_{0}+k_{S n 1} k_{01}\right)+k_{01} k_{1 n}+k_{I S C} k_{S n 1} k_{01}\right]
$$

Three-level saturation equation is expressed as follows, with $k_{T n l}=1 \mathrm{~s}^{-1}, k_{T l n}=0, k_{S n l}=1$ $\mathrm{s}^{-1}$, and $k_{l n}=0$ :

$$
S_{\text {leq__level }}=k_{T} k_{01} /\left(k_{T} k_{0}+k_{T} k_{01}+k_{\text {ISC }} k_{01}\right)
$$

Two-level saturation equation is expressed as follows, with $k_{T n 1}=1 \mathrm{~s}^{-1}, k_{T 1 n}=0, k_{S n l}=1$ $\mathrm{s}^{-1}, k_{1 n}=0, k_{T}=1 \mathrm{~s}^{-1}$, and $k_{I S C}=0$ :

$$
S_{\text {leq_2level }}=k_{01} /\left(k_{0}+k_{01}\right)
$$

For organic dyes, like Atto647N, OG488, Alexa Fluor 568, equations of five-level or four-level equilibrium are suitable. While, for NVCs or QDs, three-level and two-level equations are more suitable. Because the rate constants of $S_{n}$ and $T_{n}$ are usually much smaller than the other states, when the population probabilities of each state do not undergo any further changes within nanoseconds, the population of the excited electronic state $\left(S_{1}\right)$ can be approximated to three-level equation for either 


\section{Supporting Information}

organic dyes or inorganic markers. Furthermore, $k_{T}$ is three orders of magnitude smaller than $k_{0}$. To this end, within a laser pulse period timescale, the excited electronic steady-state equilibrium is further simplified unto two-level equation:

$$
S_{1 e q}=k_{01} /\left(k_{0}+k_{01}\right)
$$

In our simulation work, two-level and three-level equations are both suitable for research.

Competition Factor. In the following discussion, two-level equation is involved. For the excitation of single laser on the specimens, either solid beam or hollow beam, the $S_{1}$ population is as follows, $S_{\text {exc }}$ for $S_{1}$ population excited merely by solid beam and $S_{\text {hexc }}$ for $S_{1}$ population excited merely by hollow beam:

$$
\begin{gathered}
S_{\text {exc }}=k_{\text {exc }} /\left(k_{0}+k_{\text {exc }}\right) \\
S_{\text {hexc }}=k_{\text {hexc }} /\left(k_{0}+k_{\text {hexc }}\right)
\end{gathered}
$$

where $k_{\text {exc }}$ is the rate constant of $I_{\text {exc }}$ and $k_{\text {hexc }}$ for $I_{\text {hexc }}$. In pSAC modality, two beams, the hollow beam and the solid beam, are applied simultaneously. The concept of completion factor $K$ is introduced to weigh out the fluorescence completion degree between the two beams. Assume that the two beams are applied independently in the same region of interest (ROI) of the fluorescent sample. $K$ is defined as the ratio:

$$
K=S_{\text {exc }} /\left(S_{\text {exc }}+S_{\text {hexc }}\right)
$$

Actually, the two beams compete with each other concerning fluorescence emission. Thus, in pSAC, $k_{01}=k_{\text {exc }}+k_{\text {hexc }}$. The overall population of the fluorophores excited by both two beams are as follows:

$$
S_{\text {overall }}=\left(k_{\text {exc }}+k_{\text {hexc }}\right) /\left(k_{0}+k_{\text {exc }}+k_{\text {hexc }}\right)
$$

As is mentioned before, based on the rationale of pSAC, fluorescence signals excited by the sold beam is separated out via lock-in scheme, the population caused by $I_{e x c}$ and the population caused by $I_{h e x c}$ are given by the two equations:

$$
\begin{gathered}
S_{\text {exc' }}=k_{\text {exc }} /\left(k_{0}+k_{\text {exc }}+k_{\text {hexc }}\right) \\
S_{\text {hexc }}=k_{\text {hexc }} /\left(k_{0}+k_{\text {exc }}+k_{\text {hexc }}\right)
\end{gathered}
$$

After competition, the competition factor changes into the following equation:

$$
K^{\prime}=S_{\text {exc }} /\left(S_{\text {exc }}+S_{\text {hexc }}\right)
$$

The consequence of the fluorescence competition is $S_{e x c}$ which is the effective probability in $S_{l}$ of pSAC. In our discussion, fluorescence brightness equals to the excited state population. That is, $F_{\text {exc }}$ equals to $S_{\text {exc }}$, and $F_{\text {hexc }}$ equals to $S_{\text {hexc }}$. The pSAC result is expressed as follows:

$$
F_{\text {exc }}=k_{\text {exc }} /\left(k_{0}+k_{\text {exc }}+k_{\text {hexc }}\right)
$$

Photobleaching. The above discussions are all independent of bleaching concern, regardless of $k_{b}$ (like $k_{b S}$ and $k_{b T}$ ) and $k_{b n}$ (like $k_{b S n}$ and $k_{b T n}$ ). In the practical imaging experiment, especially under long-term high-power laser radiation, photobleaching plays an important role in image quality. In practical experiment, photobleaching rountinely contaminates the imaging result and decreases the SBR. The intrinsic cause of photobleaching is that the photochemical reaction takes places with activated fluorophores. ${ }^{6}$ It's especially true while molecules are in higher singlet state $S_{n}$ or higher triplet state $T_{n}$, the bleaching rate of which is normally 5-6 orders of magnitude higher than that of molecules in $S_{1}$ or $T_{1}$. In the condition of excitation with low light doses, most molecules transit between the ground state $S_{0}$ and the excited 


\section{Supporting Information}

state $S_{1}$. An extremely low proportion of molecules, about $0.4 \%$, will transit unto the triplet state $\mathrm{T}_{1}$. The $S_{0}-S_{1}$ transition dominates in case of low power radiation, the probability of which routinely could be 35 times as that of $S_{0}-S_{n}$ transition and 7 times as that of $T_{1}-T_{n}$ transition. That is, $T_{n}$ has higher bleaching rate compared with $S_{n}$. However, in the condition of high power radiation, the molecule proportion in the higher energy state could not be considered as insignificant and may have great impact on the fluorescence yield because fluorophores are in high-risk bleaching. After long-term scanning, the image quality will be greatly degraded. In order to quantatively analyze the bleaching process, bleaching rate constant $k_{z}$ is introduced in pSAC in the following equation: ${ }^{5}$

$$
k_{z}=\left(k_{b}+k_{b n} I\right) S_{\text {leq }}=\left[k_{b}+k_{b n}\left(I_{\text {exc }}+I_{\text {hexc }}\right)\right] F_{\text {exc }}
$$

The probability of photobleaching $\mathrm{p}_{\mathrm{b}}$ is also introduced to quantity the bleaching process:

$$
p_{b}=k_{z} / k_{0} S_{1 e q}=k_{b} / k_{0}+k_{b n}\left(I_{e x c}+I_{h e x c}\right) / k_{0}
$$

For negligible populations of $S_{n}$ and $T_{n}, p_{b}$ becomes a constant value $k_{b} / k_{0}$ which coincides with the conditions of three-level and two-level excitation modality. In consideration of photobleaching, after certain radiation time, $t$, the steady-state population in $S_{1}$ for $\mathrm{pSAC}$ is given by the equation:

$$
F_{\text {exc_b } b}=\left(1-e^{-k z t}\right) / p_{b}
$$

The above equation is regardless of detection efficiency and fluorescent quantum efficiency. The equation is suitable for either pulse mode or continuous-wave mode.

Pulse Delay. In order to plot out the curves including laser pulse distribution and fluorescence decay in pSAC, two-level system is involved. Fluorophores are initially in the ground state $S_{0}$ and they are excited to the excited state $S_{1}$ through photon absorption via dual-beam illumination, $I_{\text {exc }}$ and $I_{\text {hexc }}$. The spatio-temporal intensity distribution of the pulse beams is given by Equation (20): ${ }^{7}$

$$
I(x, y, t)=I_{\text {exc }}(x, y) e^{-\left(t-t_{\text {exc }}\right)^{2} / 2 T_{\text {exc }}^{2}}+I_{\text {hexc }}(x, y) e^{-\left(t-t_{\text {hexc }}\right)^{2} / 2 T_{\text {hexc }}^{2}}
$$

Where $I_{\text {exc }}(x, y)$ and $I_{\text {hexc }}(x, y)$ denote the intensity distribution of solid beam excitation and hollow beam excitation, respectively. $T_{\text {exc }}$ and $T_{\text {hexc }}$ are the pulse widths of the two beams while $t_{e x c}$ and $t_{\text {hexc }}$ are the temporal position of the two. Regardless of photobleaching impact, the population equations of the two energy levels are:

$$
\begin{gathered}
d S_{1}(x, y) / d t=\left(k_{e x c}+k_{\text {hexc }}\right) S_{0}(x, y)-k_{f} S_{1}(x, y) \\
d S_{0}(x, y) / d t=k_{f} S_{1}(x, y)-\left(k_{\text {exc }}+k_{\text {hexc }}\right) S_{0}(x, y) \\
S_{0}(x, y)+S_{1}(x, y)=1
\end{gathered}
$$

Through integrating the above equations, the time-correlated relationship is expressed as follows:

$$
S_{1}\left(x, y, t_{m+1}\right)=\left(k_{\text {exc }}+k_{\text {hexc }}\right)\left(1-e^{-\left(k_{\text {exc }}+k_{\text {hexc }}+k_{f}\right) t}\right) /\left(k_{\text {exc }}+k_{\text {hexc }}+k_{f}\right)+S_{1}\left(x, y, t_{m}\right) e^{-\left(k_{\text {exc }}+k_{\text {hexc }}+k_{f}\right) \Delta t}
$$

where $\Delta t$ denotes the increment of time scale with initial value $S_{1}(x, y, 0)=0$. For the fluorescence emitted by solid beam, the equation is changed into Equation (25):

$$
F_{\text {exc }}\left(x, y, t_{m+1}\right)=k_{\text {exc }}\left(1-e^{-\left(k_{\text {exc }}+k_{\text {hexc }}+k_{f}\right) t}\right) /\left(k_{\text {exc }}+k_{\text {hexc }}+k_{f}\right)+F_{\text {exc }}\left(x, y, t_{m}\right) e^{-\left(k_{\text {exc }}+k_{\text {hexc }}+k_{f}\right) \Delta t}
$$

With initial value $F_{\text {exc }}(x, y, 0)=0$.

Surface Plasmon Resonance. The scattering cross-section is given as:

$$
\sigma_{\text {scatter }}=8 \pi k^{4} r^{6}\left(\varepsilon_{p}-\varepsilon_{m}\right)^{2} /\left(\varepsilon_{p}+2 \varepsilon_{m}\right)^{2}
$$




\section{Supporting Information}

Where $k$ is the wave vector, $r$ is the radius of the particle, $\varepsilon_{p}$ is the dielectric constant of the particle, and $\varepsilon_{m}$ is the dielectric constant of the surrounding medium. ${ }^{8}$ Similar to electron transition process, the rate constant of GNPs can be expressed as:

$$
k_{\text {scatter }}=\sigma_{\text {scatter }} I \lambda / h c
$$

The saturation scattering intensity $I_{\text {sat }}$ is defined as the intensity when scattering cross-section is reduced to $50 \%$ of its initial and $K_{\text {scatter }}$ equals to $1 / \tau$, where $\tau$ is the lifetime of excited state of plasmon.

$$
I_{\text {sat }}=h c /\left(\lambda \sigma_{\text {scatter }} \tau\right)
$$

The attainable resolution in scattering imaging is given as follows:

$$
r_{\text {scatter }}=0.66 \lambda /\left(N A \sqrt{1+\left(I_{\text {exc }}+I_{\text {hexc }}\right) / I_{\text {sat }}}\right)
$$

Because scattering cross-section is several orders of magnitude higher than fluorescence cross-section, saturation scattering intensity could be extremely lower than fluorescence saturated intensity. To this end, in order to achieve the same resolution, the required inhibition intensity in GNP imaging by pSAC is relatively lower than that in fluorescent probe imaging. 
Supporting Information

\section{SUPPLEMENTARY FIGURES AND TABLE}

\begin{tabular}{|c|c|}
\hline Figure S1 & $\begin{array}{l}\text { Experimental system scheme of pulsed saturation competition } \\
\text { microscopy }(\mathrm{pSAC}) .\end{array}$ \\
\hline Figure S2 & $\begin{array}{l}\text { Modulation transfer function (MTF) and fluorescence intensity along } \\
\text { radial direction (either } I_{\text {exc }} \text { or } I_{\text {hexc }} \text { ) of the focal spot, as well as their } \\
\text { corresponding imaging results. }\end{array}$ \\
\hline Figure S3 & $\begin{array}{l}\text { The influence of photobleaching on the resolution and PSBR of pSAC } \\
\text { imaging in common organic dyes. }\end{array}$ \\
\hline Figure S4 & $\begin{array}{l}\text { Simulation results of dynamic intensity minimum (DyMIN) and } \\
\text { reduction of state transition cycles (RESCue) methods in pSAC. }\end{array}$ \\
\hline Figure S5 & $\begin{array}{l}\text { The influence of lateral space displacement on the resolution and PSBR } \\
\text { of pSAC imaging. }\end{array}$ \\
\hline Figure S6 & $\begin{array}{l}\text { nce of axial space displacement on the resolution and PSBR of } \\
\text { ging. }\end{array}$ \\
\hline & $\begin{array}{l}\text { The influences of hollow beam wavelength on the resolution and PSBR } \\
\text { of pSAC imaging in terms of the fluorescent dye of Rodamine } 6 \mathrm{G} \text {. }\end{array}$ \\
\hline & $\begin{array}{l}\text { Wavelength mismatch researches on varying types of fluorescent } \\
\text { microspheres. }\end{array}$ \\
\hline & $\begin{array}{l}\text { The influences of temporal displacement and pulse width on the } \\
\text { resolution of pSAC imaging. }\end{array}$ \\
\hline $\begin{array}{l}\text { Figure } \\
\text { S10 }\end{array}$ & $\begin{array}{l}\text { The fluorescence intensity simulation along radial direction and } \\
\text { fluorescence decay curve in pSAC imaging. }\end{array}$ \\
\hline $\begin{array}{l}\text { Figure } \\
\text { S11 }\end{array}$ & Supplementary experimental results on gold nanoparticles by pSAC. \\
\hline $\begin{array}{l}\text { Figure } \\
\text { S12 }\end{array}$ & $\begin{array}{l}\text { Characterizations and improvement researches on fluorescent } \\
\text { nanodiamonds. }\end{array}$ \\
\hline $\begin{array}{l}\text { Figure } \\
\text { S13 }\end{array}$ & $\begin{array}{l}\text { Supplementary experimental results of dual-color imaging of quantum } \\
\text { dots. }\end{array}$ \\
\hline Table S1 & Strength and weakness of GNPs, FNDs and QDs \\
\hline
\end{tabular}




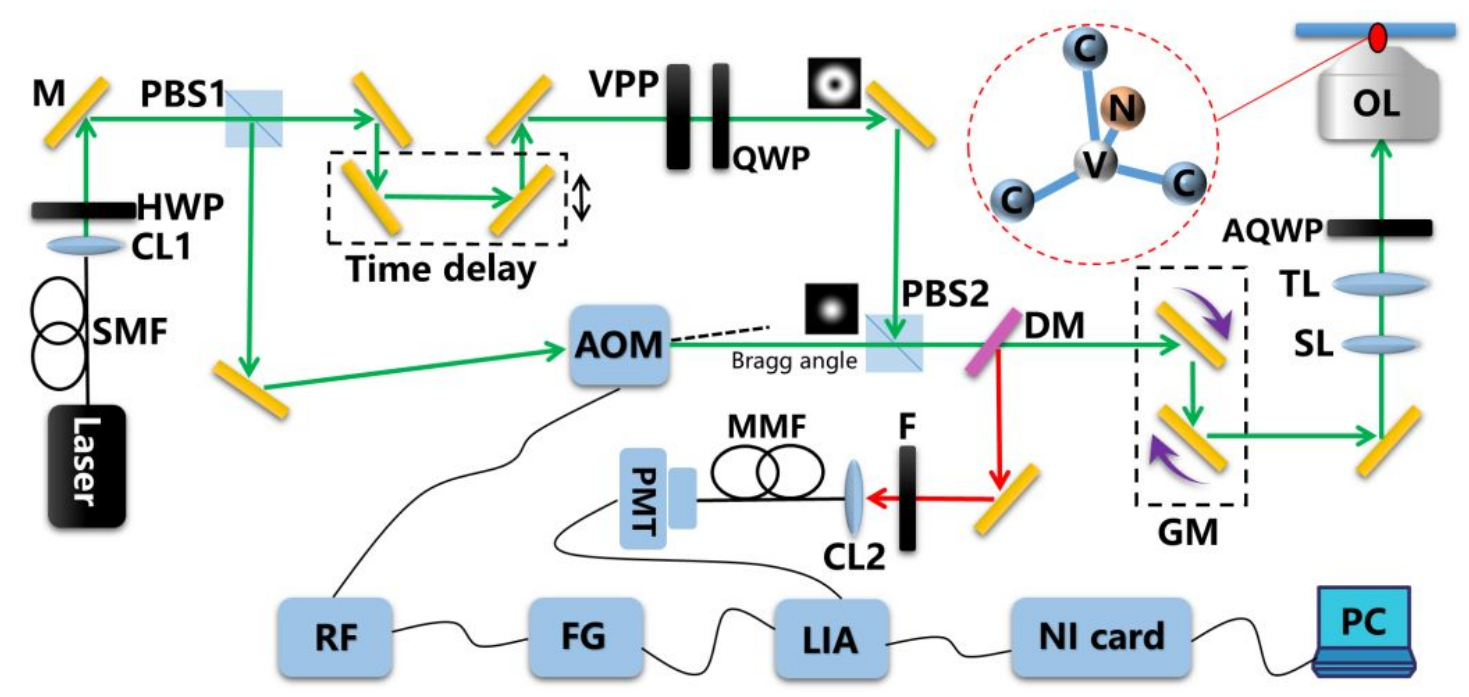

Figure S1. Experimental system scheme of pulsed saturation competition microscopy (pSAC). The following components are used. Laser: $532 \mathrm{~nm}$ laser diode, PDL 800-D, Picoquant. SMF: single-mode fibre. CL1: collimation lens. HWP: half wave plate. PBS: polarizing beam splitter. AOM: acoustic-optic modulator, 3080-125, Gooch \& Housego. M: mirror. VPP: vortex phase plate, V-532-10, Vortex Photonics. QWP: quarter wave plate. DM: dichroic mirror, ZT640rdc, Chroma. GM: galvanometer-based scanning mirrors. SL: scanning lens, SL50-CLS2, Thorlabs. TL: tube lens, TTL200MP, Thorlabs. AQWP: achromatic quarter wave plate, Thorlabs. OL: objective lens, Apo 100x/1.4 Oil, Nikon. F: filter, ET665lp, Chroma. CL2: collective lens. PMT: photomultiplier tube, H7422-40, Hamamatsu. RF: radio frequency driver. FG: function generator. LIA: lock-in amplifier, Model 7280, BRL Test, Inc. NI card: data acquisition card, NI PCI-6366, National Instruments. The black dashed square (after PBS1) shows the time delay scheme used to synchronize pulses of the solid beam and the hollow beam. Through AOM, the modulated pulse beam is deflected with a Bragg angle from the incident beam. In the red dashed circle is the basic atomic structure of nitrogen-vacancy center: three carbon atoms, one nitrogen atom substituting a carbon atom and a void replacing the position of a carbon atom. 

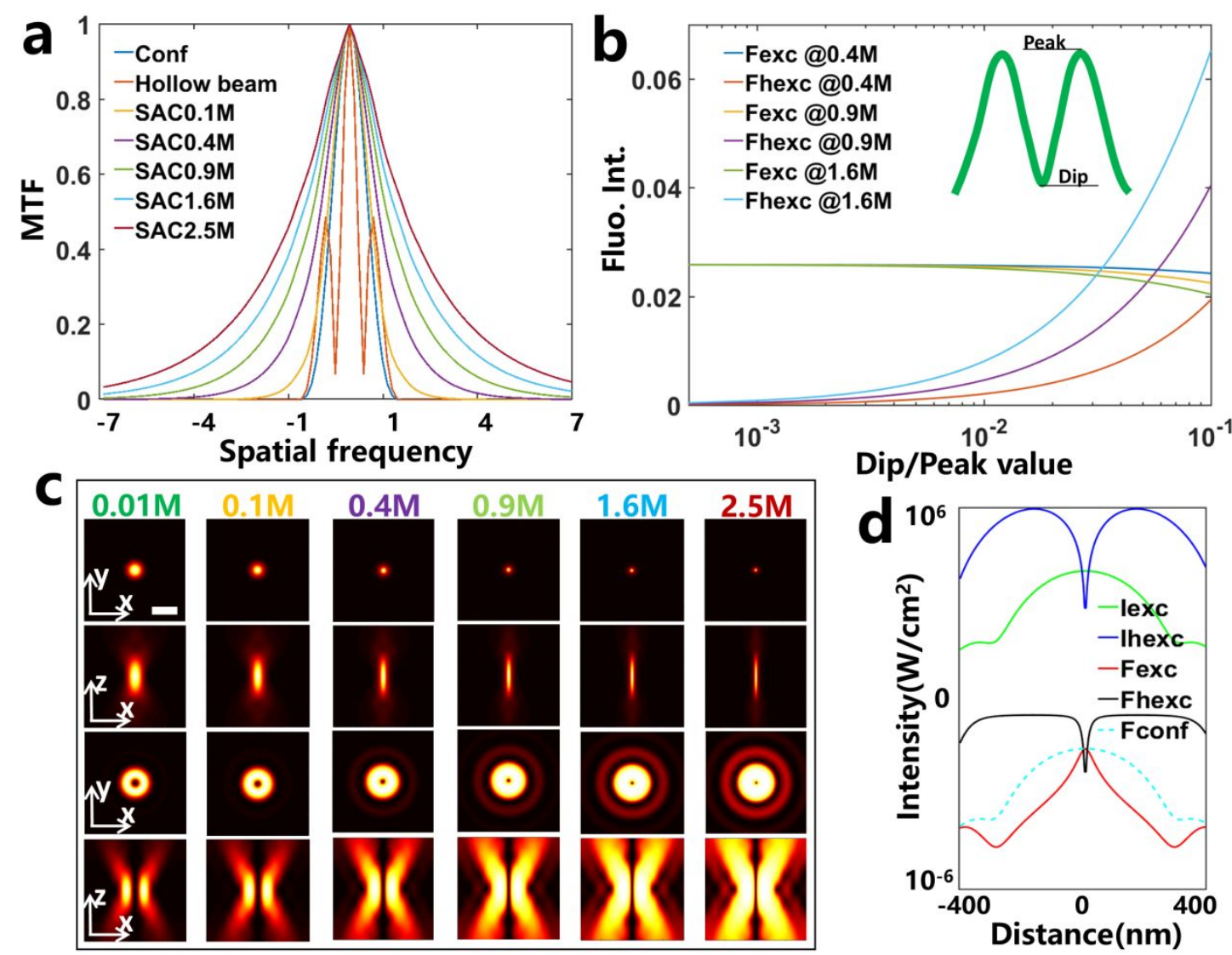

Figure S2. Modulation transfer function (MTF) and fluorescence intensity along radial direction (either $I_{\text {exc }}$ or $I_{\text {hexc }}$ ) of the focal spot, as well as their corresponding imaging results. (a) Relationship between MTF and the spatial frequency which is normalized by the value of $2 \pi N A / \lambda$. The cut-off frequency of pSAC is higher than that of confocal and can be increased with the increment of competition power $\left(I_{\text {hexc }}\right)$. (b) The impact of center imperfection of the dark spot on the fluorescence intensity in the very center area of the focal spot. The imperfection is defined as the ratio between the dip value and the peak value (as indicated by the $X$-ordinate). With the increase of dark spot imperfection from $0.01 \%$ to $10 \%, F_{\text {exc }}$ is decreased and $F_{\text {hexc }}$ is enhanced tremendously. Furthermore, higher hollow beam intensity will lead to more severe negative influence of center imperfection. (c) Numerical imaging results with varying hollow beam intensities: $0.01 \mathrm{MW} / \mathrm{cm}^{2}, 0.1 \mathrm{MW} / \mathrm{cm}^{2}, 0.4 \mathrm{MW} / \mathrm{cm}^{2}, 0.9 \mathrm{MW} / \mathrm{cm}^{2}, 1.6$ $\mathrm{MW} / \mathrm{cm}^{2}$, and $2.5 \mathrm{MW} / \mathrm{cm}^{2}$. First row of images is for $F_{\text {exc }}$ in lateral plane, second row is for $F_{\text {exc }}$ in axial plane, third row is for $F_{\text {hexc }}$ in lateral plane, and the bottom row is for $F_{\text {hexc }}$ in axial plane. Scale bar: $0.5 \mu \mathrm{m}$. (d) The laser intensity and fluorescence distribution along radial direction of the illuminated focal plane. The laser radiation power intensity is four to six orders of magnitude higher than that of fluorescence emission. $F_{\text {conf }}$ denotes the fluorescence intensity distribution along radial direction for confocal optics shown by dashed cyan line. Compared with PSF of $F_{\text {conf }}$, the PSF of the competition result $F_{\text {exc }}$ is greatly confined. 
Supporting Information
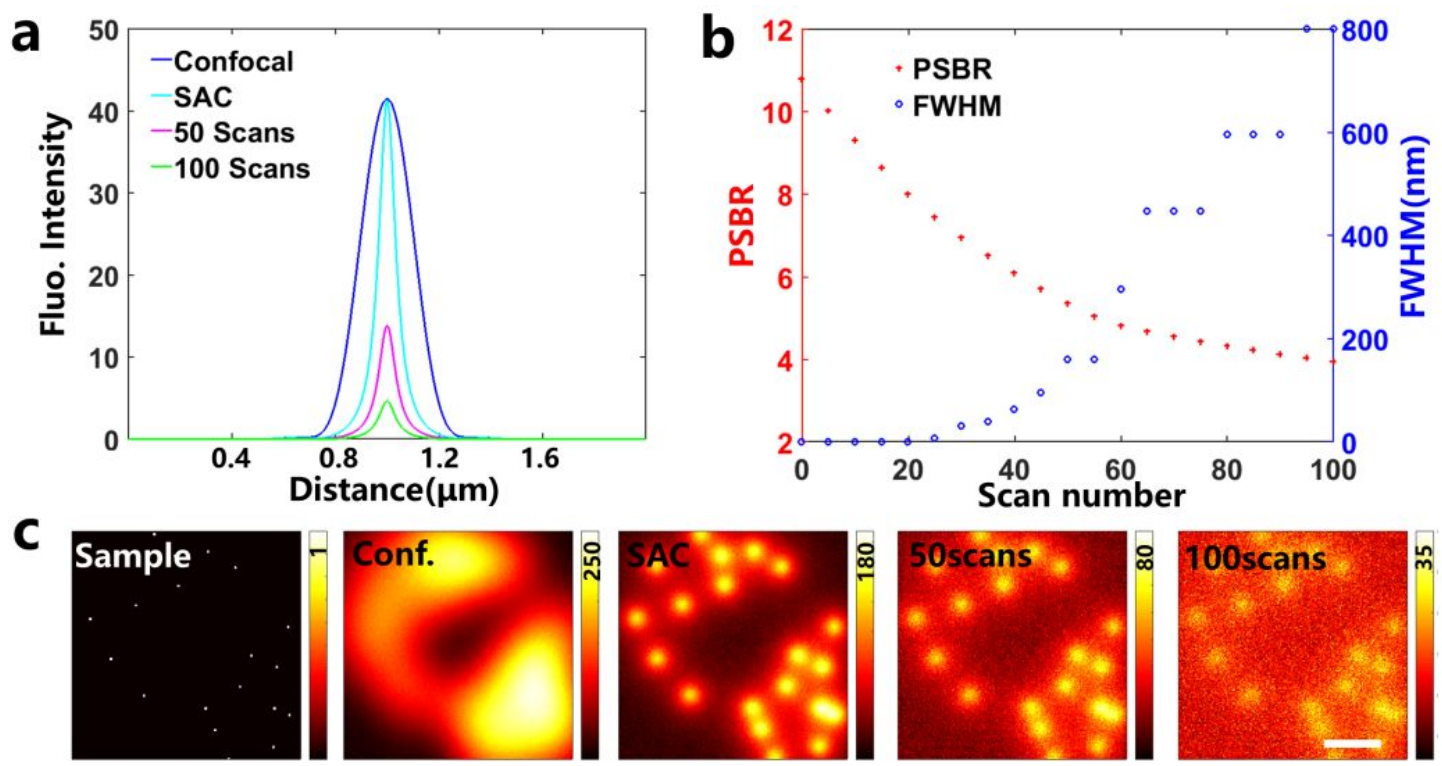

Figure S3. The influence of photobleaching on the resolution and PSBR of pSAC imaging in common organic dyes. (a) Numerical results of point spread function (PSF) of the fluorescent imaging regardless of background noise impact: confocal (blue), initial pSAC imaging (cyan), pSAC imaging after 50 scans (magenta), and pSAC imaging after 100 scans (green). The total fluorescent yields are greatly reduced after long-term illumination. (b) The illustration on the relationship between PSBR, FWHM and scan number. It's seen that the image quality is severely degraded and the attainable resolution decreases simultaneously. (c) Simulation of imaging results under varying degrees of photobleaching. The images show that background noise will have great impact on the eventual resolution. After long-term scanning, bleaching caused by high power radiation becomes an important factor for pSAC super-resolution imaging in organic probes. Scale bar: $0.5 \mu \mathrm{m}$. 
Supporting Information
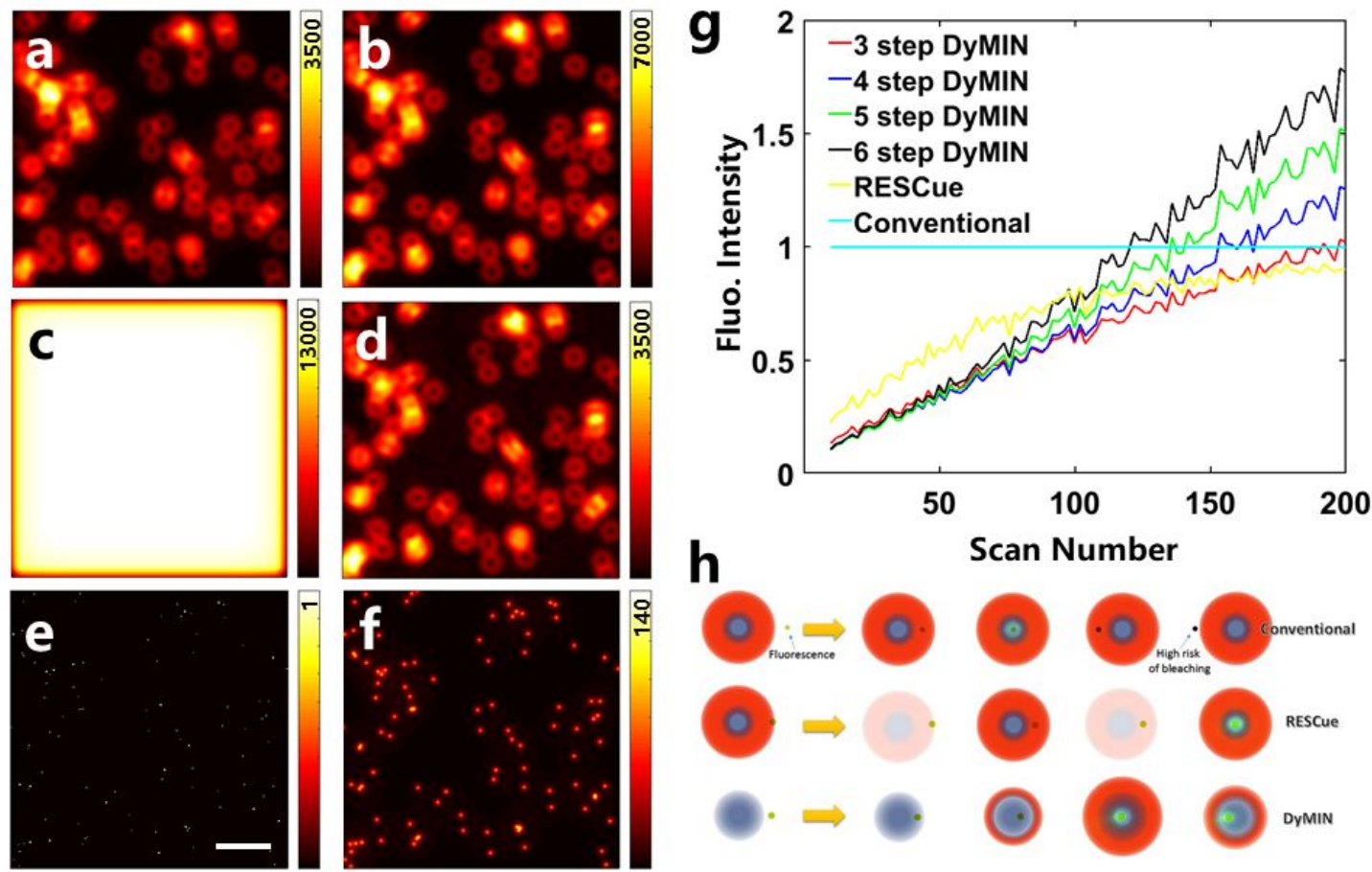

Scan Number
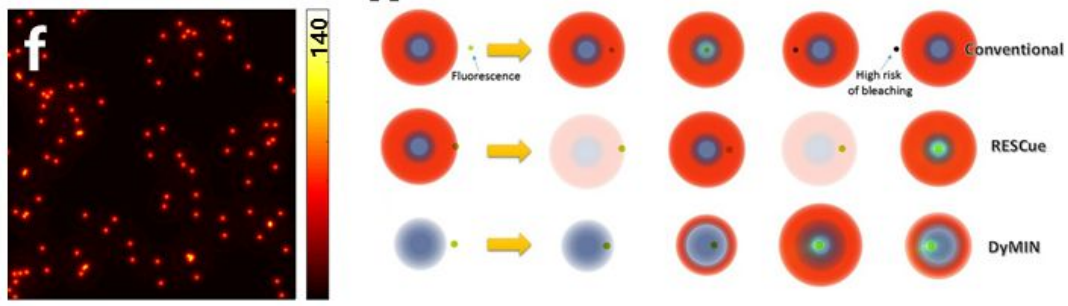

Figure S4. Simulation results of dynamic intensity minimum (DyMIN) and reduction of state transition cycles (RESCue) methods in pSAC. The two are both versatile illumination concepts for coordinate-targeted super-resolution fluorescence imaging dynamically adapting laser power in order to reduce the photobleaching. (a)-(c) Simulation results of accumulated photon counts of the applid hollow beam for DyMIN-SAC, RESCue-SAC and conventional SAC, respectively. (d) The result of (a) subtracted from (b). (e) The sample of randomly-distributed fluorescent nanoparticles. (f) Super-resolved fluorescent imaging result via pSAC. (g) Relationship between conventional microscopy and RESCue and DyMIN in varying steps. Note that we herein, for the first time to the best of our knowledge, have completed the further numerical works in 5-step and 6-step DyMIN. (h) Schematic illustration concerning the priciples between conventional microscopy and RESCue and DyMIN. ${ }^{6}$ Scale bar: $1 \mu \mathrm{m}$. 


\section{Supporting Information}
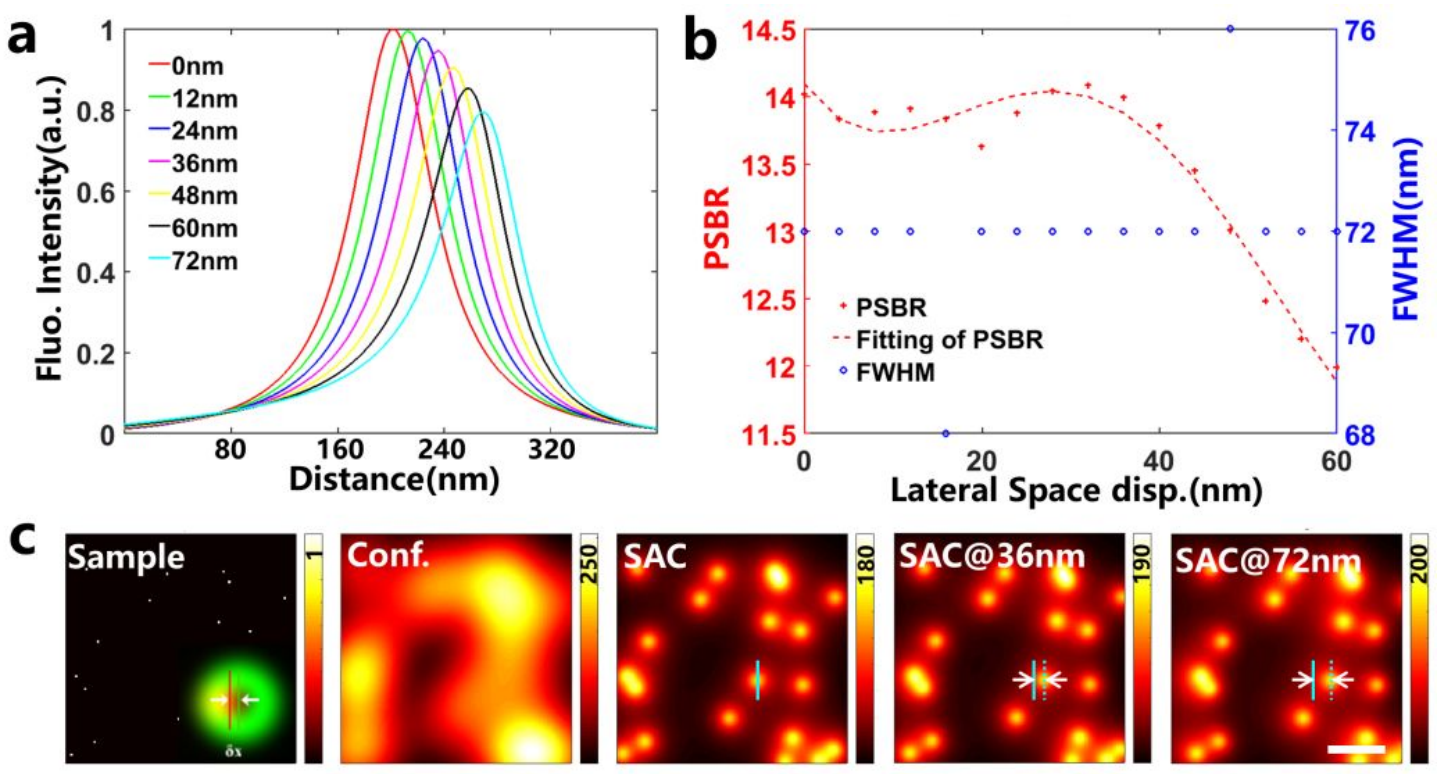

Figure S5. The influence of lateral space displacement on the resolution and PSBR of pSAC imaging. (a) PSF in cases of different spatial mismatch between the solid beam $\left(I_{\text {exc }}\right)$ and the hollow beam $\left(I_{\text {hexc }}\right)$. (b) The illustration on the relationship between PSBR, FWHM and space displacement. Note that the impact of space mismatch hardly presents obvious influence on the image quality and the resolution and FWHM is even unchanged in case of $72 \mathrm{~nm}$ mismatch. (c) Simulation of imaging results under varying degrees of spatial displacement. The cyan solid line shows the original position of one molecule and the dashed line denotes the deviated imaging of the molecule. Though the final PSBR and FWHM are almost unaffected, it is clearly seen that the whole achieved sample imaging deviates in the direction of the hollow beam inclination. Scale bar: $0.5 \mu \mathrm{m}$. 

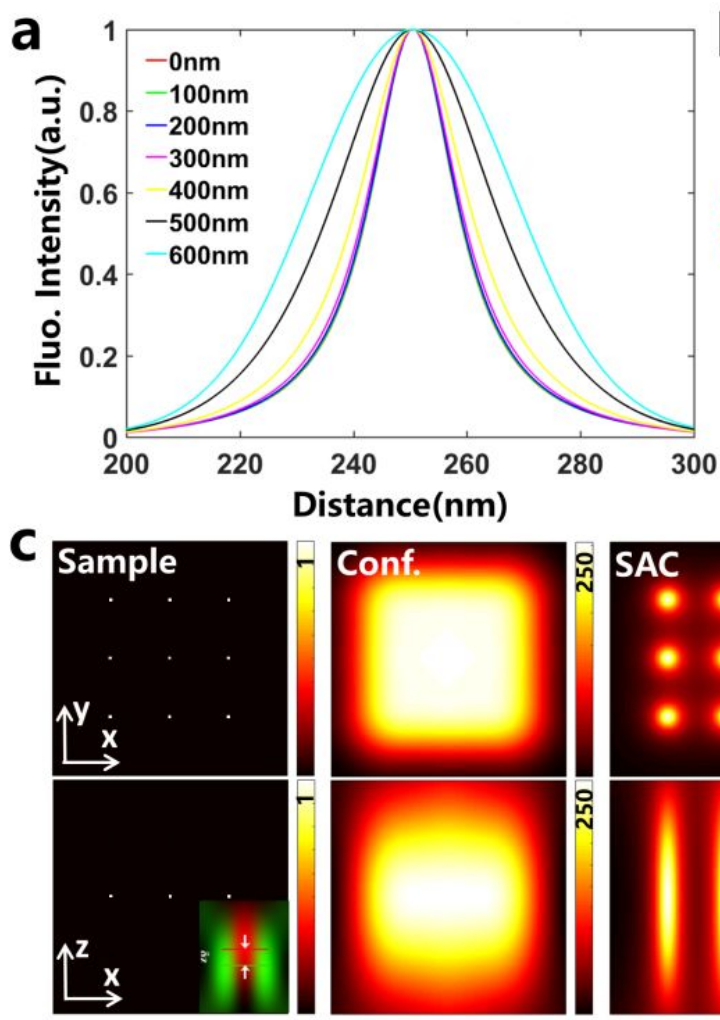
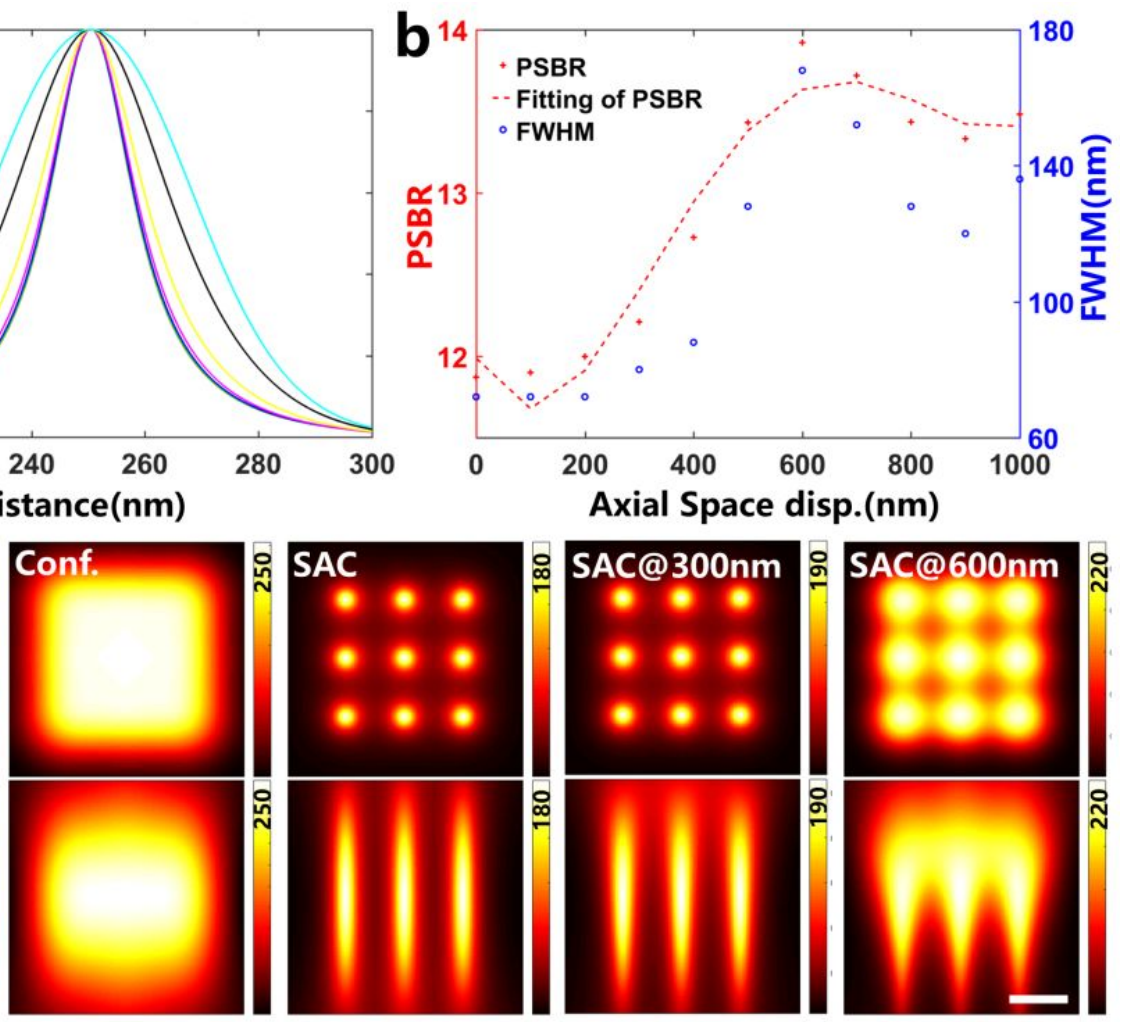

Figure S6. The influence of axial space displacement on the resolution and PSBR of pSAC imaging. (a) PSF in cases of different axial spatial mismatch (up to $600 \mathrm{~nm}$ ) between the solid beam $\left(I_{e x c}\right)$ and the hollow beam $\left(I_{\text {hexc }}\right)$ in the axial direction. The degree of mismatch lower than $300 \mathrm{~nm}$, that is, half of the excitation light wavelength or so, can be considered as negligible influence on the final lateral resolution. (b) The illustration on the relationship between PSBR, FWHM and axial space displacement. Similarly, the impact of space mismatch lower than $300 \mathrm{~nm}$ hardly presents obvious influence on the image quality and the resolution and FWHM is almost unchanged within limited axial mismatch. The above-mentioned tolerance $(300 \mathrm{~nm})$ will help to simplify the light path alignment in pSAC experiment. It is found that there exists a dramatic change in the intensity profile at around $600 \mathrm{~nm}$ defocus. It is also testified from the Figure $\mathrm{S} 2 \mathrm{c}$ (under $0.9 \mathrm{MW} / \mathrm{cm} 2$ hollow beam illumination). The radical change in $600 \mathrm{~nm}$ is the intrinsic characteristic of the vortex light field. Also, in spite of $1000 \mathrm{~nm}$ axial displacement, 120-nm lateral resolution could be achieved. (c) Simulation of imaging results under varying degrees of axial spatial displacement (upper row for $X Y$ plane and lower row for $X Z$ plane). In case of greater than $300 \mathrm{~nm}$ axial displacement, the image quality will be gradually degraded. Scale bar: $0.5 \mu \mathrm{m}$. 

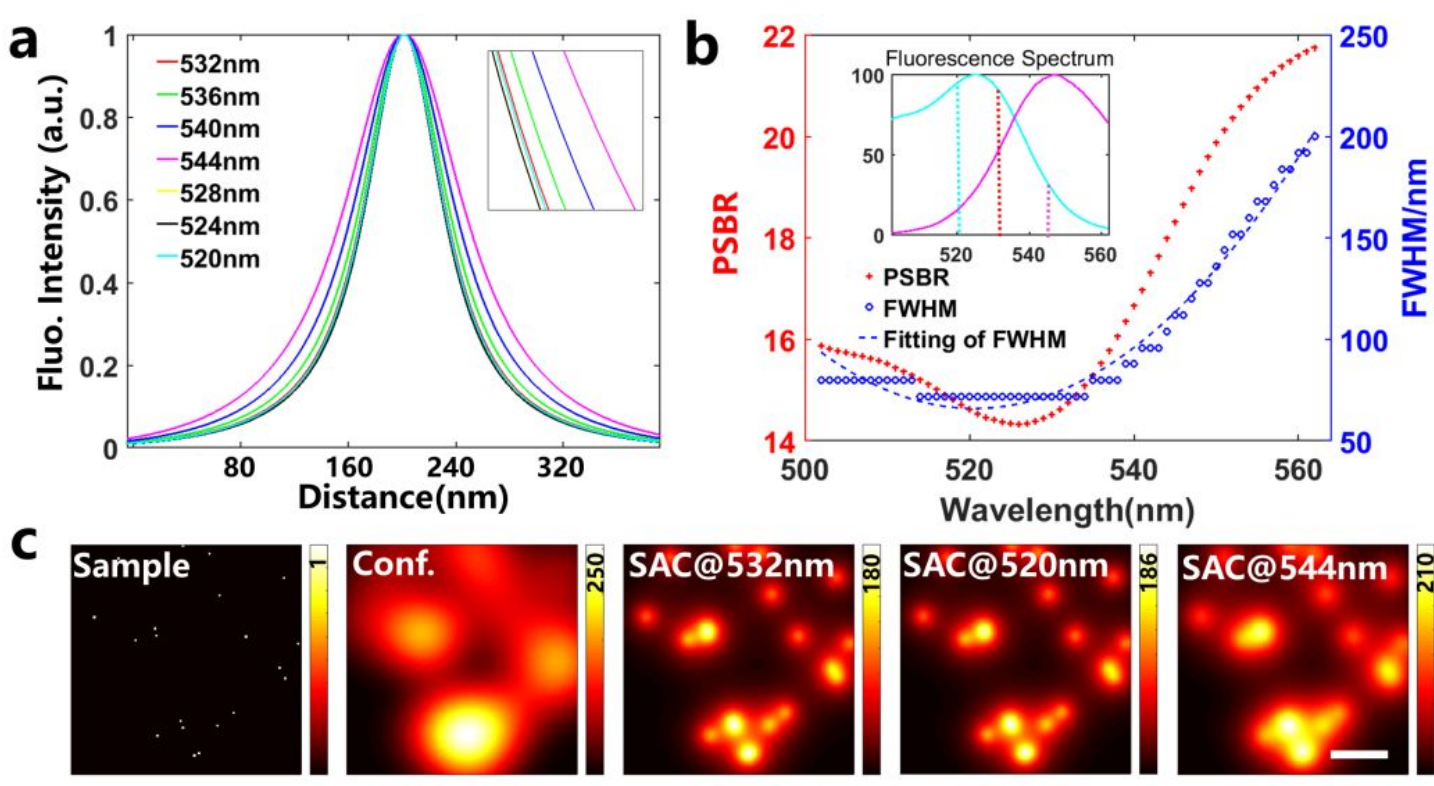

Figure S7. The influences of hollow beam wavelength on the resolution and PSBR of pSAC imaging in terms of the fluorescent dye of Rodamine 6G. (a) PSF in cases of varying wavelengths of the hollow beam $\left(I_{\text {exc }}\right) .532 \mathrm{~nm}$ and $520 \mathrm{~nm}$ exhibits highest resolution while $544 \mathrm{~nm}$ achieves the lowest resolution. Theoretically, longer wavelength of $I_{\text {hexc }}$ and higher cross-section with the excitation spectrum yields higher resolution. In the midst of the two elements, cross-section is more deterministic. (b) The illustration on the relationship between PSBR, FWHM and wavelength mismatch. Insert graph shows the fluorescence spectrum of the dye (Cyan dashed line denotes cross-section of $520 \mathrm{~nm}$ wavelength of $I_{\text {hexc }}$; red dashed line, 532 $\mathrm{nm}$ wavelength of $I_{\text {hexc }}$; magenta dashed line, $544 \mathrm{~nm}$ wavelength of $I_{\text {hexc }}$ ). Throughout the wavelength range from $520 \mathrm{~nm}$ to $532 \mathrm{~nm}$, the PSBR and FWHM change slightly and over $536 \mathrm{~nm}$ the resolution decreases. (c) Simulation of imaging results under different wavelengths of $I_{\text {hexc }}$. The image quality of $532 \mathrm{~nm}$ and $520 \mathrm{~nm}$ are almost the same which are superior to that of $544 \mathrm{~nm}$. Scale bar: $0.5 \mu \mathrm{m}$. 
Supporting Information
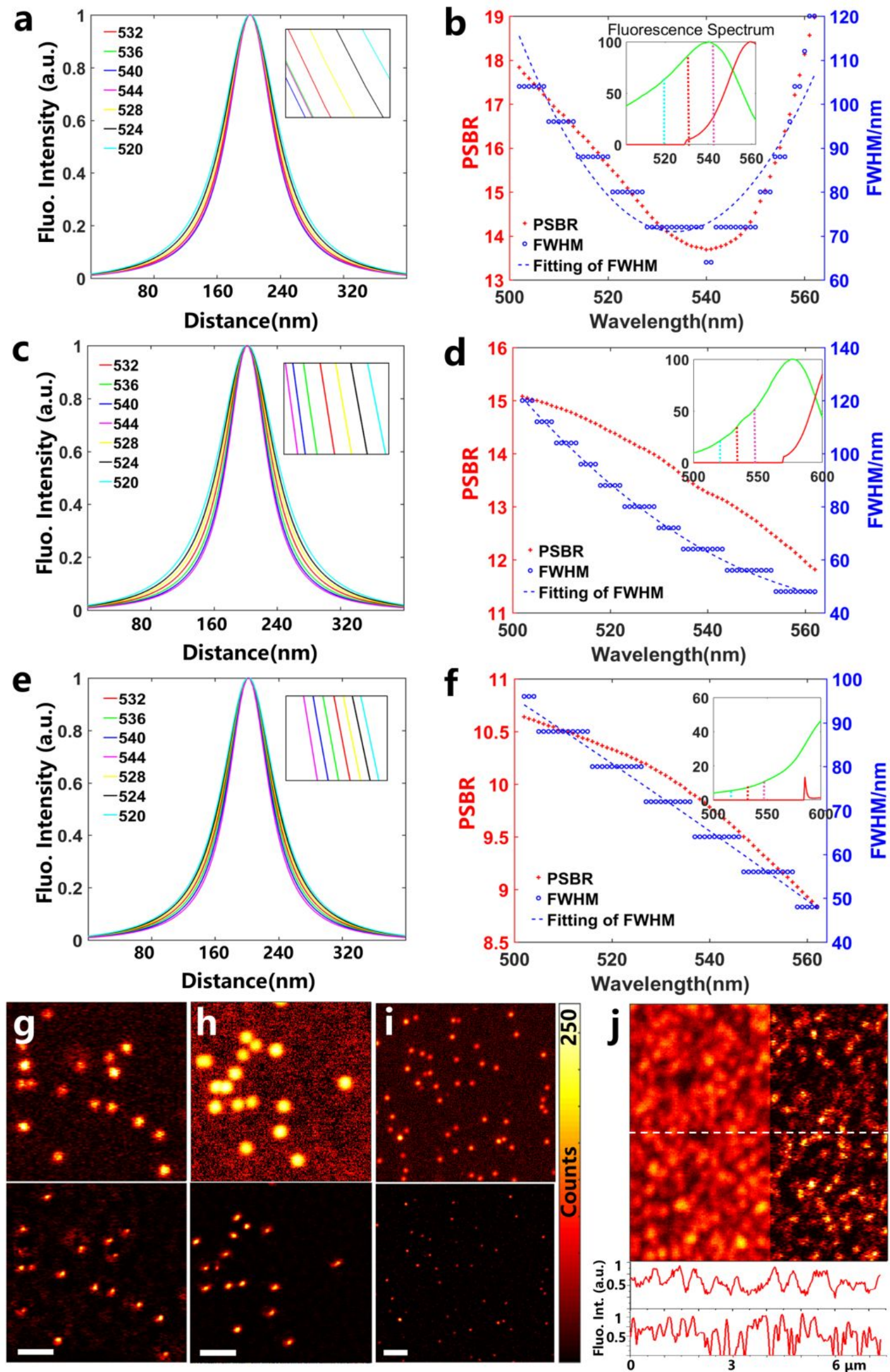

Figure S8. Wavelength mismatch researches on varying types of fluorescent microspheres. (a)-(b) Simulated wavelength mismatch investigation on the dye of 540/560 fluorescent microspheres. (b)-(c) Simulated wavelength mismatch investigation on the dye of 580/605 fluorescent microspheres. (e)-(f) Simulated 


\section{Supporting Information}

wavelength mismatch investigation on the dye of $640 / 660$ fluorescent microspheres. (g) Imaging of 540/560 fluorescent dyes (F8800, $0.1 \mu \mathrm{m}$ orange fluorescent beads, $540 / 560$, Thermofisher). The frames are $250 \times 250$ pixels, each pixel $20 \mathrm{~nm}$. Upper: confocal; Bottom: pSAC. Scale bar: $1 \mu \mathrm{m}$. (h) Imaging of 580/605 fluorescent dyes (F8801, $0.1 \mu \mathrm{m}$ red fluorescent beads, 580/605, Thermofisher). The frames are $250 \times 250$ pixels, each pixel $20 \mathrm{~nm}$. Upper: Confocal; Bottom: pSAC. Scale bar: $1 \mu \mathrm{m}$. (i) Imaging of 640/660 fluorescent dyes (N21483, $0.06 \mu \mathrm{m}$ deep red fluorescent beads, 640/660, Thermofisher). The frames are $500 \times 500$ pixels, each pixel $30 \mathrm{~nm}$. Upper: Confocal; Bottom: SAC. Scale bar: $2 \mu \mathrm{m}$. It is evident that super-resolution imaging can be realized by pSAC with lower background noise because lock-in scheme enables the decrease of irrelevant signals. (j) Imaging of 580/605 fluorescent dyes (F8770, $0.04 \mu \mathrm{m}$ red fluorescent beads, 540/560, Thermofisher). The frame is $250 \times 250$ pixels, each pixel $30 \mathrm{~nm}$. The profile plot along the dashed white line shows that pSAC improves the image SBR compared with confocal imaging. The SBR in pSAC is 2-fold greater than that in confocal imaging. In pSAC scheme, high instant peak power in pSAC inhibits the influence of background noise. Furthermore, lock-in scheme also filters some unwanted noise. 


\section{Supporting Information}
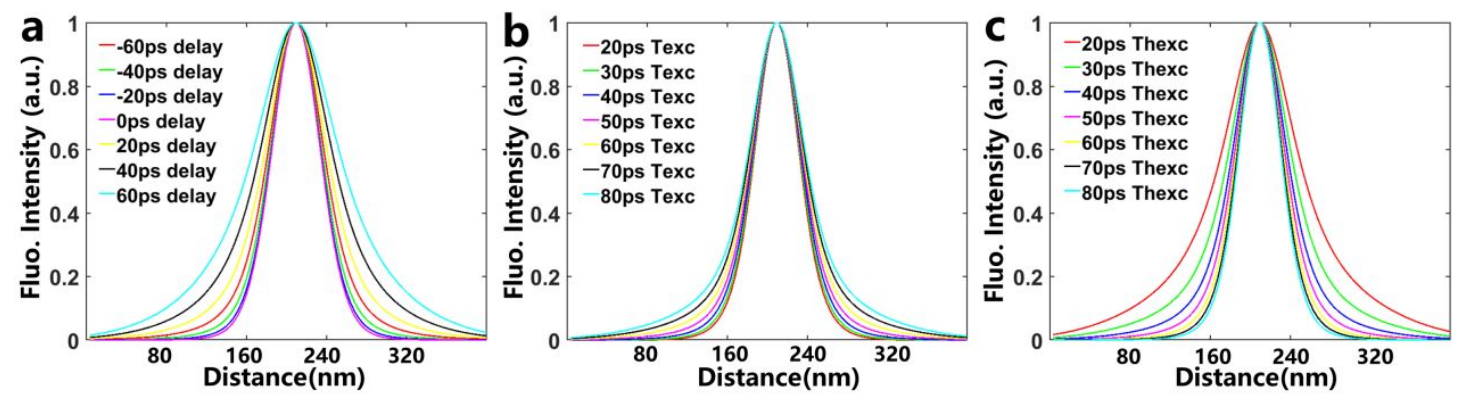

Figure S9. The influences of temporal displacement and pulse width on the resolution of pSAC imaging. ${ }^{7}$ (a) Relationships between the FWHM and $I_{\text {hexc }}$ pulse delay to $I_{\text {exc }}$ pulse. Negative value of the delay denotes to that $I_{\text {hexc }}$ pulse arrives prior to $I_{\text {exc }}$ pulse. It's worth noting that in the case that $I_{\text {hexc }}$ pulse arrives earlier than $I_{\text {exc }}$ pulse, better resolution would be achieved. It is seen that '0ps delay' obtains the highest resolution. In practical experiment, due to testing uncertainty of time-correlated single photon counting system (TCSPC) and highest temporal precision is $\sim 4 \mathrm{ps}, I_{\text {hexc }}$ pulse tends to be several picoseconds prior to $I_{\text {exc }}$ pulse. (b) Relationships between the FWHM and pulse width of $I_{\text {exc }}$. In our work, $\sim 50$ picosecond pulse width laser is implemented. The results show that shorter $I_{\text {exc }}$ relating to $I_{\text {hexc }}$ will yield higher resolution because wide $I_{\text {hexc }}$ pulse will have large probability to inhibit the fluorescence $F_{\text {exc }}$ from $I_{\text {exc }}$ pulse. (b) Relationships between the FWHM and pulse width of $I_{\text {hexc }}$. Approximately, larger $I_{\text {hexc }}$ pulse width leads to higher resolution. 

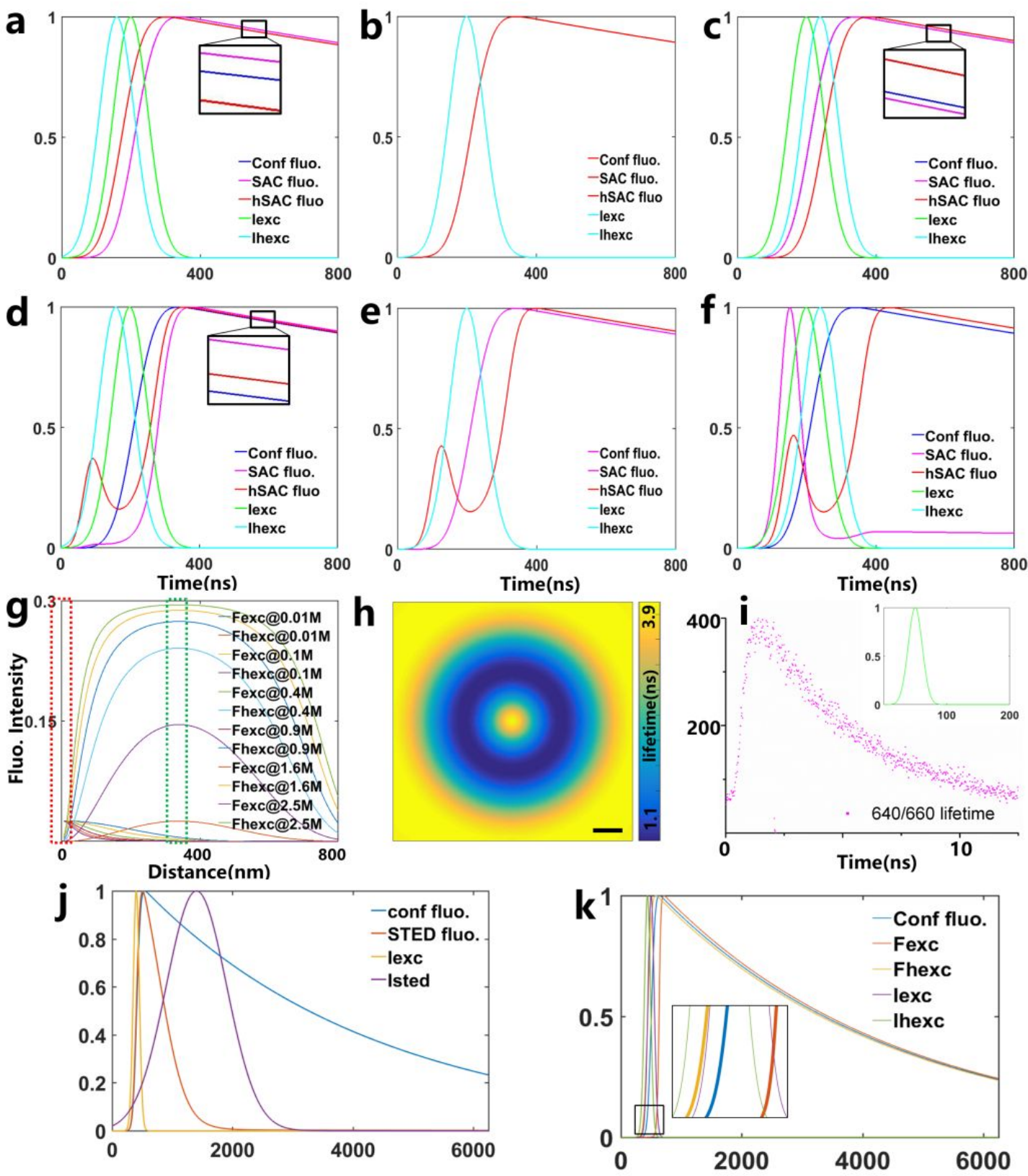

Figure S10. The fluorescence intensity simulation along radial direction and fluorescence decay curve in pSAC imaging. (a)-(c) Fluorescence decay at the center area of the focal spot in the case of -40 ps delay, 0 ps delay, and 40 ps delay, respectively, shown by red dashed square in (g). From (a) to (c), lifetime of $F_{\text {exc }}$ is almost unchanged and even equal to that of $F_{\text {hexc }}$ because in zero-intensity center part, the excitation of $I_{e x c}$ and $I_{\text {hexc }}$ are both far below the degree of fluorescence saturation. (d)-(f) Fluorescence decay at the intensity crest area of the focal spot in the case of -40 ps delay, 0 ps delay, and 40 ps delay, respectively, shown by green dashed square in (g). In intensity crest area, because of saturation effect, $F_{\text {hexc }}$ shows an emission peak at first and subsequently confined by fluorescence competition from $I_{\text {exc }}$. Eventually, the curve of $F_{\text {hexc }}$ obeys normal fluorescence decay tendency. Different from (a)-(e), in (f), the tendency of decay curve is changed greatly. That is, the lifetime of the fluorescence dyes would be decreased greatly. It is indeterministic whether the enhancement of resolution in pSAC is caused by the suppression of the lifetime or not. However, in (f), background noise is generated following the laser pulse in the timescale. Those signals include insignificant spatial information which 


\section{Supporting Information}

could be subsequently subtracted by several methods, such as time-gating, double scanning or homodyne detection. ${ }^{1,9,10}$ For (a)-(f), all $X$-ordinates denote timescale of laser pulse or fluorescence emitting and all $Y$-ordinates present the normalized fluorescence intensity. (g) Fluorescence intensity curve along radial direction of the focal spot under varying hollow beam laser intensity (ranging from $0.01 \mathrm{MW} / \mathrm{cm}^{2}$ to $\left.2.5 \mathrm{MW} / \mathrm{cm}^{2}\right)$. With the increase power of applied inhibition beam $\left(I_{\text {hexc }}\right), F_{\text {exc }}$ is gradually suppressed presenting Gaussian profile. While greater than $900 \mathrm{~kW} / \mathrm{cm}^{2}$ of $I_{\text {hexc }}, F_{\text {hexc }}$ is saturated and $F_{\text {exc }}$ is greatly confined into Lorentz distribution. This is the rationale of pSAC that could achieve super-resolution imaging. (h) Fluorescence lifetime simulation of (f) in the focal area implemented by two beams. The above simulation is implemented with $I_{\text {exc }} 10 \mathrm{~kW} / \mathrm{cm}^{2}$ and $I_{\text {hexc }} 900 \mathrm{~kW} / \mathrm{cm}^{2}$ in Rodamine 6G. Scale bar: $1 \mu \mathrm{m}$. (i) Experimental result of the fluorescence decay during a single laser pulse period ( $80 \mathrm{MHz}$ repetition frequency). The $Y$-ordinate denotes the fluorescence intensity (unit: kilo counts). The top-right insert graph shows excitation pulse of 50 ps width (denoted by the green curve) in which the $X$-ordinate is the timescale (unit: ps) and $Y$-ordinate is normalized laser intensity. The experiment is employed in $60 \mathrm{~nm}$ fluorescent particles $(0.06 \mu \mathrm{m}$ deep red fluorescent beads, 640/660, Thermofisher). The analysis result shows that the lifetime of the fluorophores is $3.61 \mathrm{~ns}$. (j) The simulated fluorescence decay curve of STED. (k) The simulated fluorescence decay curve of pSAC. Repetition frequency: $80 \mathrm{MHz}$. Pulse width of excitation beam and competition beam: $50 \mathrm{ps}$. Depletion pulse width: $500 \mathrm{ps}$. In STED, depletion pulse is 800 ns delay to excitation pulse. In pSAC, the hollow excitation beam pulse is $60 \mathrm{ps}$ prior to the solid excitation pulse. 


\section{Supporting Information}
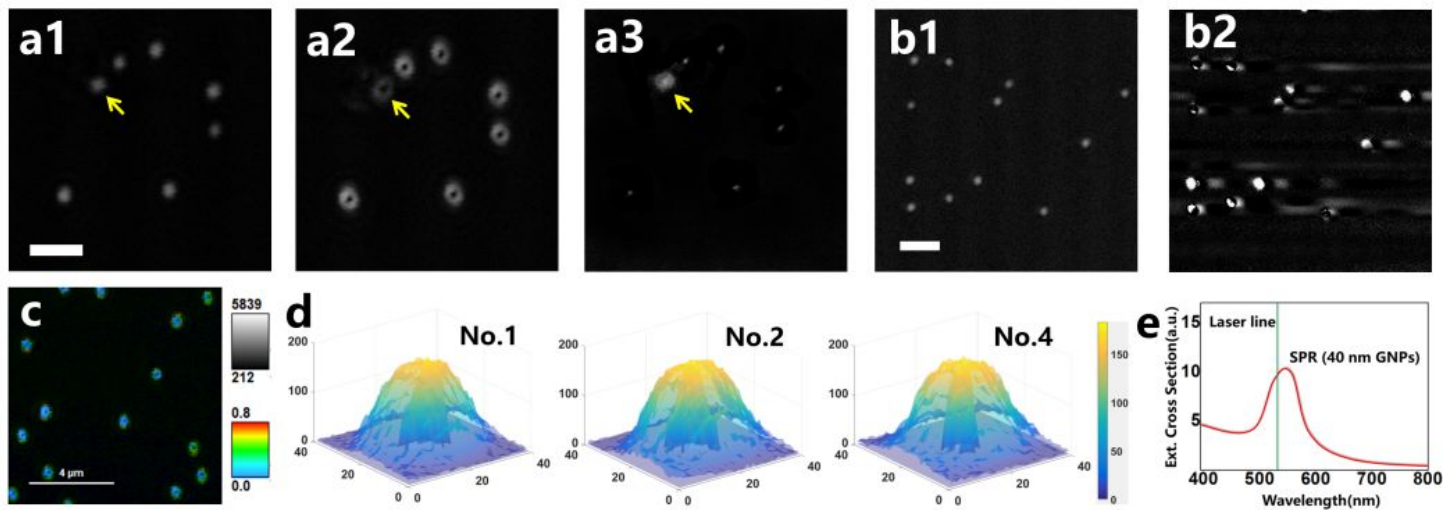

Figure S11. Supplementary experimental results on gold nanoparticles by pSAC. (a1)-(a3) Imaging result of $40 \mathrm{~nm}$ gold nanoparticles, by confocal, hollow confocal and pSAC, respectively. The region of interest (ROI) denoted by the yellow arrow may exist more than one particles such that the signals of which overlap and thereby the resolution improvement is compromised. Scale bar: $2 \mu \mathrm{m}$. (b1) Confocal imaging on gold nanoparticles. (b2) Failure acquisition of the sub-diffraction image. Because the time constant is too low $(20 \mu \mathrm{s})$, incomplete signal demodulation deteriorates the image quality, yielding distortion of signals. Scale bar: $1 \mu \mathrm{m}$. (c) The lifetime analysis on gold nanoparticles. The gray bar denotes the received photon counts and the color bar stands for lifetime distribution in the fluorescence lifetime microscopy (FLIM) experiment. (d) The 3D numerical analysis of other three nanoparticles in Figure 3. The $X$ - and $Y$ - ordinates denote the pixel numbers (pixel size $10 \mathrm{~nm}$ ) and the $Z$-ordinate stands for fluorescent signal intensity. (e) The dependency of SPR of 40 nm GNPs on excitation wavelength. The $Y$-ordinate is the extinction cross-section. Our experimental laser wavelength locates close to the peak SPR. 

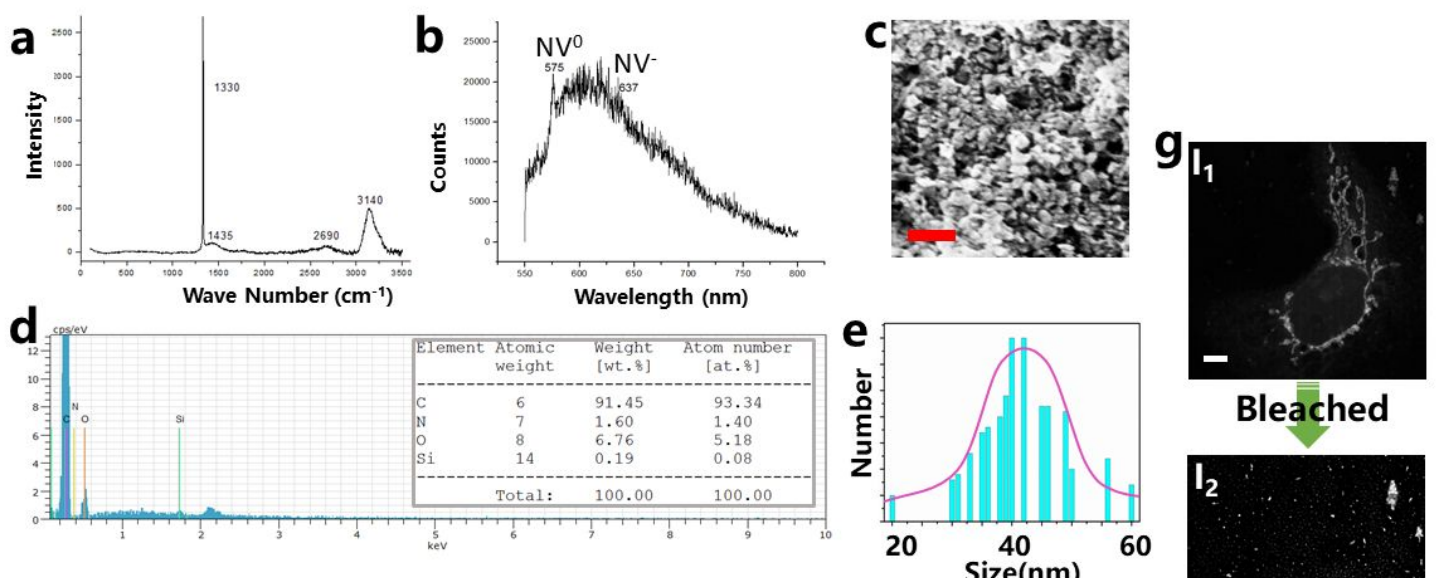

Bleached
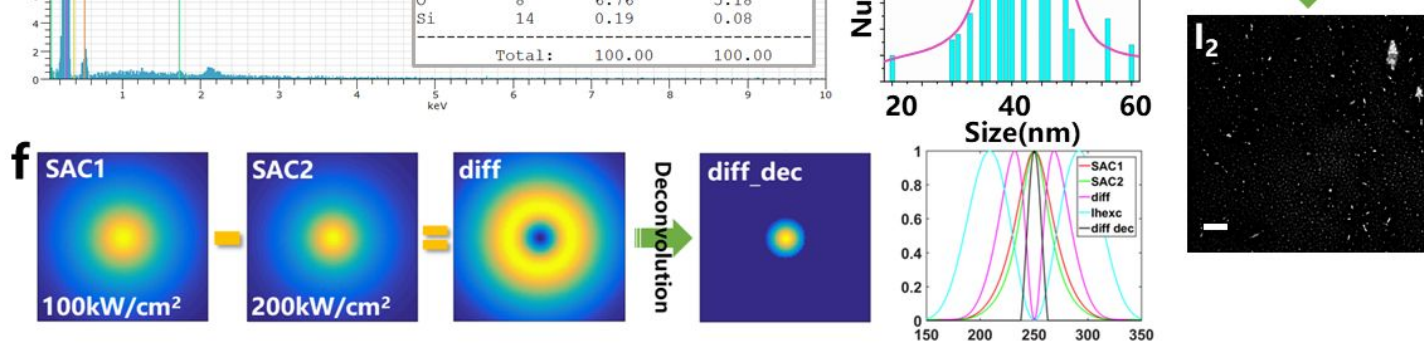

Figure S12. Characterizations and improvement researches on fluorescent nanodiamonds. (a) Raman analysis of NVCs. The fluorescent nanodiamonds (FNDs) are acquired commercially (Adams nanotechnologies, Inc.). The values, 1330 and 2690, denote diamond peak and its double frequency peak, respectively. The value 3140 denotes graphene double frequency peak, indicating that there exist sp2-hybridized structures on the FND crystal surface. The data is obtained on ThermoFisher DXR532 using $532 \mathrm{~nm}$ laser. (b) Photoluminescence test of NVCs. Both neutral electronic nitrogen-vacancy centers $\left(\mathrm{NV}^{0}\right)$ and negative electronic nitrogen-vacancy centers $\left(\mathrm{NV}^{-}\right)$are observed. The data is obtained on home-built confocal spectrum system using $405 \mathrm{~nm}$ lasers. (c) Electron scanning imaging of aggregated fluorescent nanodiamond particles. Scale bar: $1 \mu \mathrm{m}$. (d) The energy dispersion spectrum (EDS) test of the FND sample. Additional element oxygen is due to oxidation of carbon element on the diamond crystal surface. Silicon is the impurity, the percentage of which is within the tolerable limit and is negligible to experiment works. The data is obtained on the field emission scanning electron microscope (SEM, Zeiss Sigma 300). (e) FND particle size distribution analysis in (c). The average size of the particles is $\sim 40 \mathrm{~nm}$. (f) The rationale of differential saturated absorption competition algorithm (dSAC) which is able to further increase the final resolution through subtraction and deconvolution operation. SAC1 is operated with $I_{\text {hexc }} 100 \mathrm{~kW} / \mathrm{cm}^{2}$ and SAC2 is implemented with $I_{\text {hexc }} 200 \mathrm{~kW} / \mathrm{cm}^{2}$. The curve comparison shows that the FWHM of the acquired PSF is even smaller than that of $\mathrm{SAC1}$ or SAC2. (g) Before bleaching, the two kinds of fluorescent signals are detected $\left(I_{1}\right)$. After bleaching, only signals from FNDs are detected $\left(I_{2}\right)$. By subtracting $I_{2}$ from $I_{1}$, fluorescent signals from Mitotracker Deep Red is obtained $\left(I_{3}=I_{1}-I_{2}\right)$. Through combination of $I_{2}$ and $I_{3}$, dual-color imaging based on photobleaching is achieved. Scale bar: $5 \mu \mathrm{m}$. 


\section{Supporting Information}

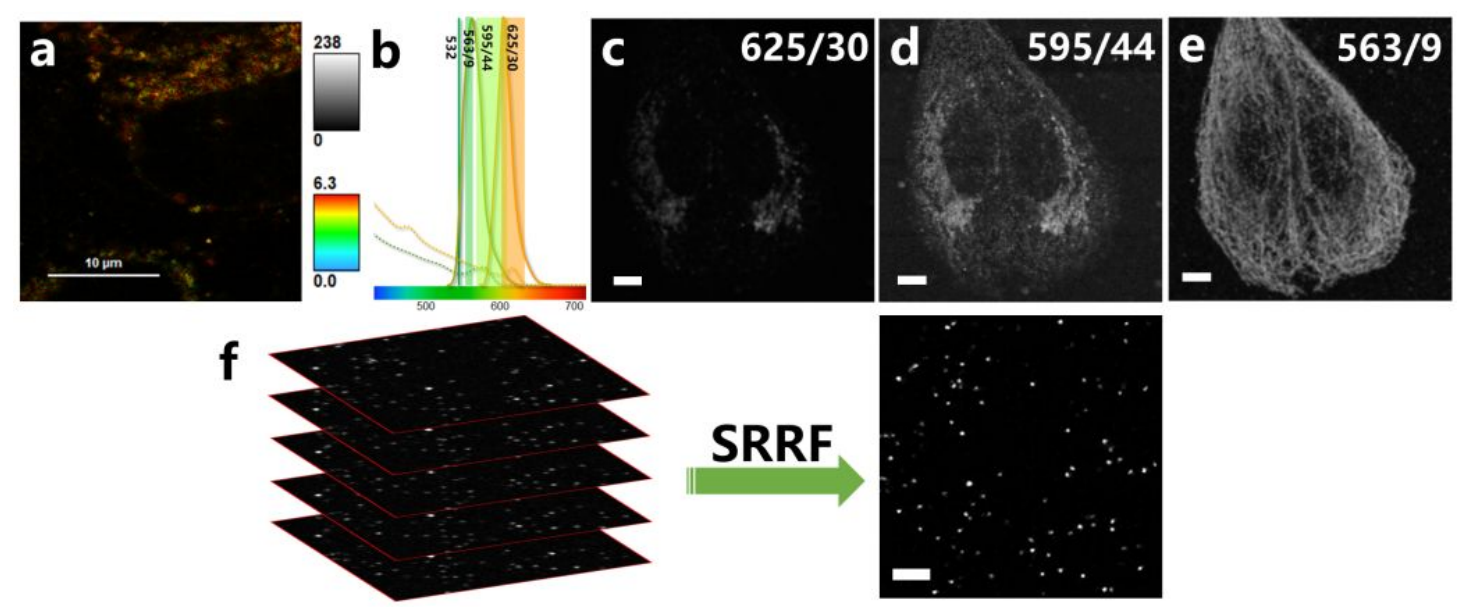

Figure S13. Supplementary experimental results of dual-color imaging of quantum dots. (a) Fluorescent lifetime microscopy (FLIM) result. The gray bar denotes the received photon counts and the color bar stands for lifetime distribution in FLIM. (b) Fluorescence spectrum. (c)-(e) In order to reduce the influence of fluorescent crosstalk, an extra filtering window is added. There are three several filtering windows: 563/9 (green window), 595/44 (middle window), and 625/30 (red window). It is evident that red window have the crosstalk with Qdot565 emission spectrum and thus the middle window is introduced to balance the fluorescent signals in red window. Background fluorescence caused by crosstalk in red window is subtracted according to the microtubule signal proportion between green window and the middle window. (f) Application of super-resolution radial fluctuation microscopy (SRRF) in quantum dots. Using the features of blinking of QDs, by reconstruction of 100 sequential frames, the resulting resolution is enhanced. In future pSAC research, this algorithm holds great potential to be used in resolution enhancement of biological structures conjugated with QDs, like microtubule and mitochondria. 


\section{Supporting Information}

Table S1. Strength and weakness of GNPs, FNDs and QDs

\begin{tabular}{|c|c|c|c|}
\hline & $\mathbf{G N P}^{2,4,8,11}$ & FND $^{12-14}$ & $\mathbf{Q D}^{15-18}$ \\
\hline \multirow{7}{*}{ Strength } & High photostability & High photostability & High photostability \\
\hline & High contrast & Photomagnetic property & High quantum yield \\
\hline & Size-dependent SPR & Mechanical stability & Narrow emission spectrum \\
\hline & Excellent biocompatibility & Excellent biocompatibility & Excellent biocompatibility \\
\hline & Large cross-section* & Chemical stability & Atomic dimensions \\
\hline & & Electroconductivity & Size-dependent emission \\
\hline & & High thermal conductivity & \\
\hline Weakness & $\begin{array}{l}\begin{array}{l}\text { Aggregation-induced red } \\
\text { shift }^{4}\end{array} \\
\end{array}$ & Relatively low yield ${ }^{19}$ & Blinking ${ }^{20}$ \\
\hline
\end{tabular}

*The cross-section of GNPs is routinely 6-7 orders of magnitude higher than that of fluorescent dyes. 


\section{REFERENCES}

1. Vicidomini, G.; Schoenle, A.; Ta, H.; Han, K. Y.; Moneron, G.; Eggeling, C.; Hell, S. W., STED Nanoscopy with Time-Gated Detection: Theoretical and Experimental Aspects. Plos One 2013, 8 (1), e54421.

2. Ouyang, X.; Qin, F.; Ji, Z.; Zhang, T.; Xu, J.; Feng, Z.; Yang, S.; Cao, Y.; Shi, K.; Jiang, L.; Li, X., Saturation Scattering Competition for Non-fluorescence Single-wavelength Super-resolution Imaging. APL Photonics 2018, 3 (11), 110801.

3. Enderlein, J., Breaking the Diffraction Limit with Dynamic Saturation Optical Microscopy. Appl. Phys. Lett. 2005, 87 (9), 094105.

4. Chu, S. W.; Su, T. Y.; Oketani, R.; Huang, Y. T.; Wu, H. Y.; Yonemaru, Y.; Yamanaka, M.; Lee, H.; Zhuo, G. Y.; Lee, M. Y.; Kawata, S.; Fujita, K., Measurement of a Saturated Emission of Optical Radiation from Gold Nanoparticles: Application to an Ultrahigh Resolution Microscope. Phys. Rev. Lett. 2014, 112 (1), 017402.

5. Eggeling, C.; Volkmer, A.; Seidel, C. A. M., Molecular Photobleaching Kinetics of Rhodamine $6 \mathrm{G}$ by One- and Two-photon Induced Confocal Fluorescence Microscopy. Chemphyschem 2005, 6 (5), 791-804.

6. Li, C.; Liu, S.; Wang, W.; Liu, W.; Kuang, C.; Liu, X., Recent research on stimulated emission depletion microscopy for reducing photobleaching. Journal of Microscopy 2018, 271 (1), 4-16.

7. Gao, P.; Prunsche, B.; Zhou, L.; Nienhaus, K.; Nienhaus, G. U., Background Suppression in Fluorescence Nanoscopy with Stimulated Emission Double Depletion. Nature Photon. 2017, 11 (3), 163-170.

8. Chu, S.-W.; Wu, H.-Y.; Huang, Y.-T.; Su, T.; Lee, H.; Yonemaru, Y.; Yamanaka, M.; Oketani, R.; Kawata, S.; Shoji, S.; Fujita, K., Saturation and Reverse Saturation of Scattering in a Single Plasmonic Nanoparticle. ACS Photon. 2014, 1 (1), 32-37.

9. Ye, S.; Yan, W.; Zhao, M.; Peng, X.; Song, J.; Qu, J., Low-Saturation-Intensity, High-Photostability, and High-Resolution STED Nanoscopy Assisted by CsPbBr3 Quantum Dots. Adv. Mater. 2018, 30 (23), e1800167.

10. Ronzitti, E.; Harke, B.; Diaspro, A., Frequency dependent detection in a STED microscope using modulated excitation light. Opt. Express 2013, 21 (1), 210-219.

11. Xu, J.; Zhang, T.; Yang, S.; Feng, Z.; Li, H.; Hu, D.; Qin, F.; Ouyang, X.; Cao, Y.; Jiang, L.; Li, X., Plasmonic Nanoprobes for Multiplexed Fluorescence-free Super-Resolution Imaging. Adv. Optical Mater. 2018, 6 (1800432), 1-8.

12. Jelezko, F.; Wrachtrup, J., Single defect centres in diamond: A review. Phys. Status Solidi 2010, 203 (13), 3207-3225.

13. Hui, Y. Y.; Hsiao, W. W. W.; Haziza, S.; Simonneau, M.; Treussart, F.; Chang, H. C., Single Particle Tracking of Fluorescent Nanodiamonds in Cells and Organisms. Curr. Opin. Solid. St. M. 2017, 21 (1), 35-42.

14. Schirhagl, R.; Chang, K.; Loretz, M.; Degen, C. L., Nitrogen-Vacancy Centers in Diamond: Nanoscale Sensors for Physics and Biology. Annu. Rev. Phys. Chem. 2014, 65, 83-105.

15. Michalet, X.; Pinaud, F. F.; Bentolila, L. A.; Tsay, J. M.; Doose, S.; Li, J. J.; 


\section{Supporting Information}

Sundaresan, G.; Wu, A. M.; Gambhir, S. S.; Weiss, S., Quantum Dots for Live Cells, in Vivo Imaging, and Diagnostics. Science 2005, 307 (5709), 538-544.

16. Medintz, I. L.; Uyeda, H. T.; Goldman, E. R.; Mattoussi, H., Quantum dot bioconjugates for imaging, labelling and sensing. Nature Materials 2005, 4 (6), 435-446.

17. Hoshino, A.; Manabe, N.; Fujioka, K.; Suzuki, K.; Yasuhara, M.; Yamamoto, K., Use of fluorescent quantum dot bioconjugates for cellular imaging of immune cells, cell organelle labeling, and nanomedicine: surface modification regulates biological function, including cytotoxicity. J. Artificial Organs 2007, 10 (3), 149-157.

18. Xing, Y.; Rao, J., Quantum dot bioconjugates for in vitro diagnostics \& in vivo imaging. Cancer Biomarkers 2008, 4 (6), 307-319.

19. Jin, D.; Xi, P.; Wang, B.; Zhang, L.; Enderlein, J.; van Oijen, A. M., Nanoparticles for super-resolution microscopy and single-molecule tracking. Nat Methods 2018, 15 (6), 415-423.

20. Ji, B.; Giovanelli, E.; Habert, B.; Spinicelli, P.; Nasilowski, M.; Xu, X.; Lequeux, N.; Hugonin, J.-P.; Marquier, F.; Greffet, J.-J.; Dubertret, B., Non-blinking Quantum Dot with a Plasmonic Nanoshell Resonator. Nature Nanotech. 2015, 10 (2), 170-175. 